\title{
Longitudinal dynamics with rf phase modulation in the Brazilian electron storage ring
}

\author{
N.P. Abreu* \\ Instituto de Física "Gleb Wataghin," Universidade Estadual de Campinas, C.P. 6165, 13083-970 Campinas, Brazil, \\ and LNLS, Laboratório Nacional de Luz Síncrotron, Caixa Postal 6192, CEP 13083-970, Campinas, Brazil \\ R. H. A. Farias and P.F. Tavares \\ LNLS, Laboratório Nacional de Luz Síncrotron, Caixa Postal 6192, CEP 13083-970, Campinas, Brazil
}

(Received 11 October 2006; published 12 December 2006)

\begin{abstract}
In the Brazilian synchrotron light source (LNLS-Laboratório Nacional de Luz Síncrotron), we observed that modulating the phase of the accelerating fields at approximately twice the synchrotron frequency suppressed remarkably well a longitudinal coupled-bunch mode of the beam driven by a higher order mode in one of the radiofrequency (rf) cavities. In this work, we present the results of a set of systematic measurements, in single and multibunch mode, aimed at characterizing the effects of rf phase modulation on the beam. We compare those experiments with the results of tracking simulations and of a theoretical model in which Landau damping is the stabilizing mechanism that explains the suppression of the longitudinal coupled-bunch instability. We also measure the frequency of the stable islands created in longitudinal phase space by phase modulation and the longitudinal beam transfer function as a function of the modulation frequency and amplitude. The experimental results are in good agreement with theoretical expectations.
\end{abstract}

DOI: 10.1103/PhysRevSTAB.9.124401

PACS numbers: 29.20.Lq, 29.27.-a

\section{INTRODUCTION}

Studies related to the effects of phase or amplitude modulation of the accelerating radiofrequency (rf) fields in circular accelerators date back to the early 1990's when a series of classical experiments were performed with the aim of understanding basic aspects of nonlinear longitudinal beam dynamics at low current in hadron machines [15]. Apart from the fundamental interest of those experiments, related to the possibility of direct observations of nonlinear phenomena such as the formation of islands in phase space and measurement of synchrotron tune variations with amplitude, the main applications envisaged by those works were related to issues such as lifetime limitations due to rf noise, the development of superslow extraction techniques, and the use of parametric feedback for multibunch instabilities. Those early works laid the basic theoretical foundations for subsequent interest in applying rf phase or voltage modulation to electron storage rings used as synchrotron light sources [6,7] as a tool to control electron bunch density [8,9], increase beam lifetime [10], and reduce the amplitude or even suppress coupled-bunch instabilities [10-12], resulting in relevant improvement to the performance of those machines. Some of those previous works analyzed the effects of rf phase modulation at the first and third harmonics of the synchrotron frequency $[10,13,14]$, whereas others (such as the present paper) [11] focus on modulation at the second harmonic.

*Electronic address: natalia@lnls.br
However, in spite of the success of the implementation of such techniques at several laboratories, some aspects of the physical mechanisms that explain the effectiveness of the reduction of the amplitude of coupled-bunch modes (CBMs) are still not completely understood and a quantitative theoretical description is lacking. There is also some difficulty in simulating the effects of phase modulation in multibunch mode; most of the work done so far simulates a single bunch and it is not obvious how to infer the multibunch behavior from these results.

All experiments reported here were conducted at the LNLS $1.37 \mathrm{GeV}$ electron storage ring [15], which routinely operates to produce synchrotron radiation for users. The original motivation for our interest in rf phase modulation was the need to circumvent longitudinal instabilities that were observed in the LNLS ring right after the installation of a second rf cavity by the end of 2003 [16]. This cavity has a longitudinal higher order mode (HOM) (with a frequency of $903 \mathrm{MHz}$ ) which caused an intermittent orbit distortion that mimics the second order dispersion function and which appeared when the beam coupled with the HOM of the cavity exciting a large longitudinal dipolar oscillation. With temperature and plunger scans we were able to identify the longitudinal mode L1 (associated with the CBM No. 133) of the new rf cavity as the main source of instabilities in the machine. Since it was not possible to find a cavity operational condition that would be free from instabilities, an active solution in the form of phase modulation of the rf fields at approximately twice the synchrotron frequency was attempted with success, having a 
noticeable impact on CBM amplitudes and helping to alleviate the orbit fluctuation.

In this work, we develop a theoretical model and a simulation tool to analyze the effects of phase modulation on the longitudinal beam dynamics. According to our model, rf phase modulation enhances stability through the increase of the incoherent frequency spread that results as the electrons populate the phase-space islands created by the action of the modulation. In order to verify the model and simulation results, we perform a set of systematic measurements to characterize the effects of modulation on the beam dynamics observing directly the island tunes, the increased coherent damping time, and the changes to the longitudinal electron beam distribution caused by the modulation.

The paper is organized as follows. We begin (Sec. II) with the motivation that led us to introduce rf phase modulation routinely in user shifts at the LNLS storage ring and with a report of our attempts to stabilize the beam after the installation of the new rf cavity. In Sec. III we calculate the electron equations of motion when subjected to phase modulation of the rf field at the second harmonic of the synchrotron frequency using the Hamiltonian formalism and, using the Fokker-Planck equation, we derive the particle distribution in phase space. Also in Sec. III we calculate the dynamics for small oscillations around the stable fixed points created when modulation is turned on and, with the standard formalism used to calculate Landau damping, we find the increase in the amount of damping of centroid oscillations related to phase modulation. In this section we also calculate the longitudinal beam transfer function and the stability diagram showing that the stable area is increased when phase modulation is turned on. In Sec. IV we present results of simulations of the effects of phase modulation in single bunch and multibunch modes. In those simulations, one of the circulating bunches has an internal structure whereas all others are treated as macroparticles. Finally, in Sec. V, we compare the results of theory and simulation with experiments in which we measure the island structure, the frequency for small oscillations around the stable fixed points created by rf phase modulation, the increase in the amount of damping due to modulation, and the longitudinal beam transfer function (BTF), verifying the results obtained with the theoretical model presented in Sec. III. Conclusions are given in Sec. VI.

\section{CBM STABILIZATION BY PHASE MODULATION}

The LNLS synchrotron light source is based on a 1.37 GeV electron storage ring and operates with $250 \mathrm{~mA}$ of initial current in normal users shifts. A summary of the main parameters for the Brazilian machine is shown in Table I. At the end of 2003, the rf system of the ring was upgraded and a new cavity was installed $[16,17]$.
TABLE I. LNLS ring main parameters.

\begin{tabular}{|c|c|c|}
\hline Parameter & Symbol & Value \\
\hline Beam energy & E & $1.37 \mathrm{GeV}$ \\
\hline Natural energy spread & $\sigma_{\epsilon} / E$ & $5.4 \times 10^{-4}$ \\
\hline Circumference & $C$ & $93.252 \mathrm{~m}$ \\
\hline rf frequency & $f_{\mathrm{rf}}$ & $476.066 \mathrm{MHz}$ \\
\hline Harmonic number & $h$ & 148 \\
\hline Momentum compaction factor & $\alpha$ & $8.3 \times 10^{-4}$ \\
\hline Radiation loss per turn & $U_{0}$ & $114 \mathrm{keV}$ \\
\hline Synchronous phase $^{\mathrm{a}}$ & $\phi_{s}$ & $166.8^{\circ}$ \\
\hline Synchrotron frequency ${ }^{a}$ & fs & $26 \mathrm{kHz}$ \\
\hline
\end{tabular}

${ }^{\mathrm{a} C a l c u l a t e d}$ using $V_{\mathrm{rf}}=500 \mathrm{kV}$.

This second rf cavity was added to the system in order to prepare it for power demands that the future installation of insertion devices will bring about. After operation with two cavities was initiated, the beam suffered from frequent instability outbreaks during user runs.

The typical signature of the beam instability is an orbit fluctuation measured only in the horizontal plane, as shown in Fig. 1. The distortion pattern has the same 6-fold symmetry of the storage ring and shows up in the form of a fast transition between two states. For most of the user run periods, small orbit fluctuations with amplitudes of $\pm 3 \mu \mathrm{m}$ were observed and could be detected at the most sensitive beam lines. However, larger amplitude distortions were sometimes observed as shown in Fig. 1(a).

The origin of the orbit fluctuations was identified as being related to large amplitude dipolar longitudinal oscillations caused by the interaction of the beam with HOMs excited in the cavities. In fact, the second order term of the nonlinear dispersion function $\eta_{1}(s)$ was measured and has the same profile observed in the beam position monitors (BPMs) reading and shown in Fig. 1(b). When a longitudinal dipolar oscillation is present due to the influence of a $\mathrm{HOM}$, the relative energy deviation is given by $\delta(t)=$ $\delta_{0} \cos \Omega_{s} t$. The closed orbit distortion is then

$$
x_{\epsilon}(s, t)=\delta(t) \eta(s)+\delta^{2}(t) \eta_{1}(s) .
$$

Since the oscillation is fast (the synchrotron frequency $\Omega_{s}$ is of the order of $25 \mathrm{kHz}$ ) the BPMs measure only average positions

$$
\left\langle x_{\epsilon}\right\rangle=\frac{1}{2} \eta_{1}(s) \delta_{0}^{2},
$$

whereas the maximum amplitude of the oscillations is given by

$$
x_{\epsilon}^{\max }(s)=\delta_{0} \eta(s)=\sqrt{\frac{2\left\langle x_{\epsilon}\right\rangle}{\eta_{1}(s)}} \eta(s) .
$$

$x_{\epsilon}^{\max }$ shows up in the detectors of the beam lines as an effective increase of the horizontal beam size. The same orbit distortion can be obtained by phase modulating (at the 

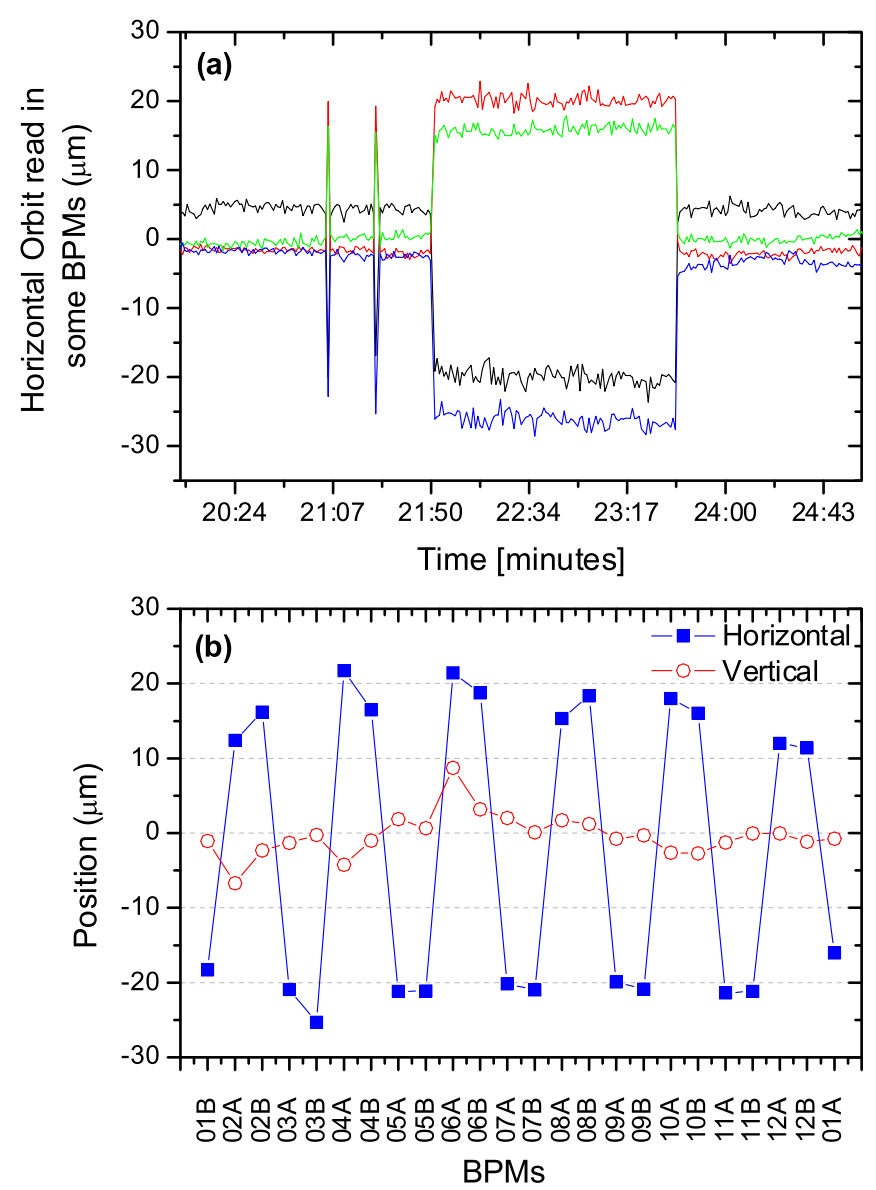

FIG. 1. (Color) Orbit distortion caused by a longitudinal CBM. The distortion occurs only in the horizontal orbit and has the same 6-fold symmetry of the machine. (a) Time evolution of the horizontal orbit measured by four different BPMs during a user shift showing the orbit fluctuation caused by the instability excited by a HOM. (b) Profile of the orbit fluctuation along the storage ring. This is obtained by subtracting the orbit right before the fluctuation from the orbit right after it.

synchrotron frequency) the rf master generator that drives the rf stations.

Attempts to eliminate the instabilities by changing the parameters of both cavities have proved to be not very effective. The search for a convenient operational point of the cavity was performed by scanning the parameters that can be used to control the cavity tuning (temperature, plunger position and axial deformation) and measuring the corresponding HOM frequencies.

The identification of the potentially harmful modes was possible by comparing them with the modes measured for a similar cavity used in our injector booster synchrotron, which had been fully characterized in the laboratory, and by comparing the frequency shifts of the modes with those predicted from their simulated field maps. The procedure adopted to analyze the effect of the HOMs is the same used for similar cavities operating at ELETTRA and ANKA [18] and consisted in the determination of the growth rates

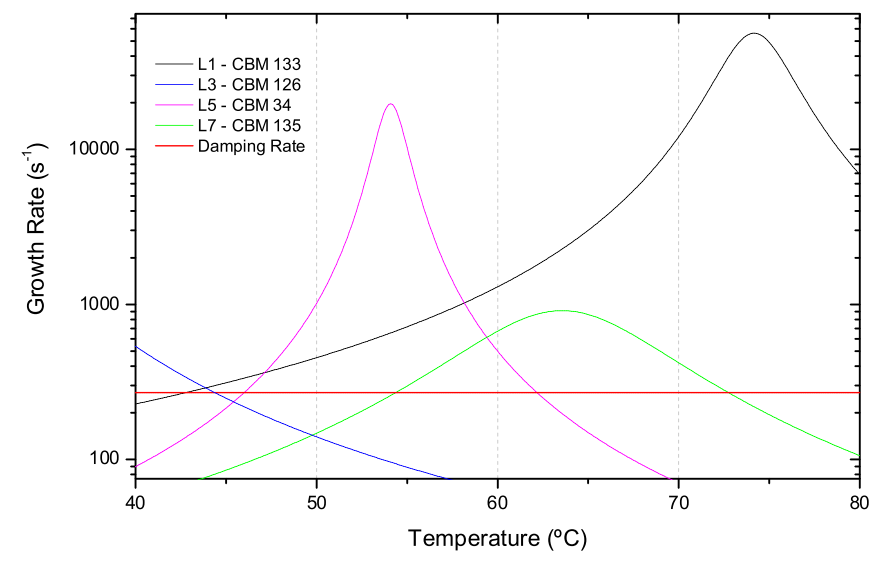

FIG. 2. (Color) CBM growth rates for the new rf cavity in the best operation point for the plunger position, calculated for $E_{0}=$ $1.37 \mathrm{GeV}$ and $I=250 \mathrm{~mA}$. Notice the absence of a mode free region.

of the coupled-bunch mode (CBM) instabilities as a function of temperature and plunger positions. By mapping the variation of the modes with these parameters, we determined the regions for which the growth rates of the oscillations are below the radiation damping rate, which for the LNLS storage ring at nominal operating energy is $1 / \tau_{\text {rad }} \approx$ $270 \mathrm{~s}^{-1}$, as shown in Fig. 2.

The results of the HOM survey showed a comfortable situation for the old cavity. The temperature range for which there are no dangerous longitudinal HOMs is quite wide. Unfortunately, the same is not true for the new cavity. A list of some of its main longitudinal modes is shown in Table II. $T_{C}$ is the critical temperature of the mode, defined as the temperature at which the frequency of the HOM matches the frequency of the CBM oscillation. The values of $Q_{L}$ have been measured in the new cavity but those of $R_{s} / Q_{0}$ are simulated values. The main problem with the new cavity is the longitudinal mode L1 whose critical temperature is just at the edge of the available temperature control range but is rather insensitive to changes of the cavity tuning parameters, as shown in Fig. 2. An attempt was made to shift that mode using the plunger already installed in the cavity but the extent of the plunger motion was not enough to accomplish the task [17].

The coupled-bunch mode associated with the L1 mode, CBM No. 133, is always present in the beam spectrum except in those regions where the growth rate of other

TABLE II. Main $\mathrm{TM}_{0 m n}$ modes of cavity 2 .

\begin{tabular}{lcrrrr}
\hline \hline Mode & $\begin{array}{c}f \\
(\mathrm{MHz})\end{array}$ & $\begin{array}{c}R_{s} / Q_{0} \\
(\Omega)\end{array}$ & \multicolumn{1}{c}{$Q_{L}$} & $\begin{array}{c}T_{C} \\
\left({ }^{\circ} C\right)\end{array}$ & $\begin{array}{c}c \\
\text { CBM }\end{array}$ \\
\hline L1 & 904.128 & 29.7 & 20000 & 74 & 133 \\
L3 & 1356.89 & 5.4 & 4000 & 31 & 126 \\
L5 & 1538.25 & 9.5 & 4000 & 54 & 34 \\
L9 & 2040.125 & 8.2 & 25000 & 28 & 43 \\
\hline \hline
\end{tabular}




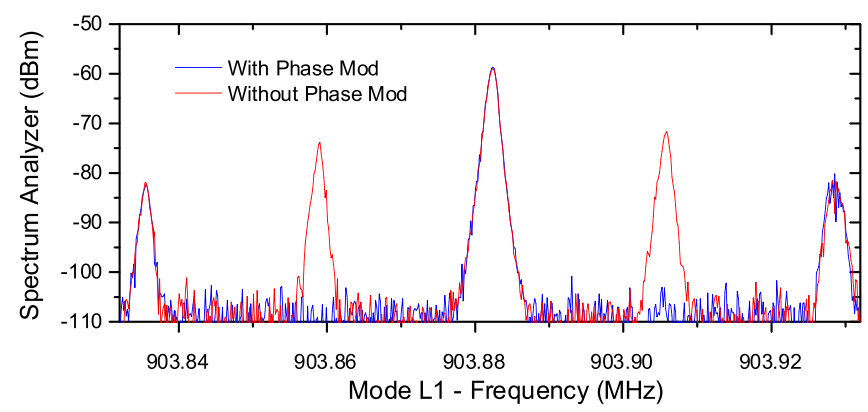

FIG. 3. (Color) Measured frequency spectrum of the signal from a button pickup in the storage ring demonstrating the suppression of dipolar synchrotron sidebands as a result of rf phase modulation at the second harmonic of the synchrotron frequency.

modes, such as CBM No. 126 associated to mode L3, is larger. HOM mapping was useful for the identification of the best operation point but this point turned out to be not good enough. As a result, an active solution in the form of phase modulation of the rf fields at approximately twice the synchrotron frequency was attempted with remarkable success. Figure 3 shows the longitudinal spectrum of the stored beam as measured by a button pickup. The suppression of the dipolar synchrotron sideband of the harmonic No. 281 of the revolution frequency by more than $40 \mathrm{~dB}$ illustrates the damping of this CBM by $\mathrm{rf}$ phase modulation.

\section{THEORETICAL MODEL}

\section{A. Longitudinal Hamiltonian with phase modulation}

We initially follow the standard Hamiltonian analysis for the longitudinal dynamics with phase modulation [14] in which we expand the complete Hamiltonian near the second harmonic resonance of the synchrotron frequency and retain only the lower order terms. The Hamiltonian for an electron in a bunch subjected to phase modulation can be written as follows:

$$
\begin{aligned}
H(\delta, \phi ; t)= & \frac{\omega_{s} \delta^{2}}{2}+\omega_{s} \tan \bar{\phi}_{s}\left[\sin \phi \cos \left(A_{m} \sin \omega_{m} t\right)\right. \\
& \left.+\cos \phi \sin \left(A_{m} \sin \omega_{m} t\right)\right] \\
& -\omega_{s} \cos \phi \cos \left(A_{m} \sin \omega_{m} t\right) \\
& +\omega_{s} \sin \phi \sin \left(A_{m} \sin \omega_{m} t\right)-\omega_{s} \phi \tan \bar{\phi}_{s},
\end{aligned}
$$

where $\omega_{s}=2 \pi f_{s}$ is the synchrotron frequency, $\bar{\phi}_{s}=\pi-$ $\phi_{s}$ where $\phi_{s}$ is the synchronous phase, $A_{m}$ is the modulation amplitude, and $\omega_{m}$ is the modulation frequency. Using the action-angle variables $(J, \psi)$ we make the following transformation:

$$
\begin{aligned}
& \delta=-\sqrt{2 J} \sin \left(\psi+\omega_{m} t / 2+\pi / 4\right), \\
& \phi=\sqrt{2 J} \cos \left(\psi+\omega_{m} t / 2+\pi / 4\right),
\end{aligned}
$$

which describes the trajectories of the particles in phase space near the second harmonic resonance in a reference frame which rotates at half of the modulation frequency. The resulting time-averaged Hamiltonian $K$ [Appendix A] is then

$$
\langle K\rangle_{t}=\left(\omega_{s}-\frac{\omega_{m}}{2}\right) J-\frac{\omega_{s} J^{2}}{16}+\frac{\omega_{s} \epsilon J}{4} \cos 2 \psi,
$$

where $\epsilon=A_{m} \tan \bar{\phi}_{s}$

The fixed points of the system are the solutions of

$$
\frac{d J}{d t}=-\frac{\partial K}{\partial \psi}=0 \quad \text { and } \quad \frac{d \psi}{d t}=\frac{\partial K}{\partial J}=0
$$

and are

$$
J_{S F P}= \begin{cases}8\left(1-\frac{\omega_{m}}{2 \omega_{s}}\right)+2 \epsilon, & \omega_{m} \leq(2+\epsilon / 2) \omega_{s} \\ 0, & \omega_{m}>(2+\epsilon / 2) \omega_{s}\end{cases}
$$

corresponding to the angles $\psi_{S F P}=0, \pi$, which are stable since $\cos 2 \psi>0$, and the unstable fixes points are
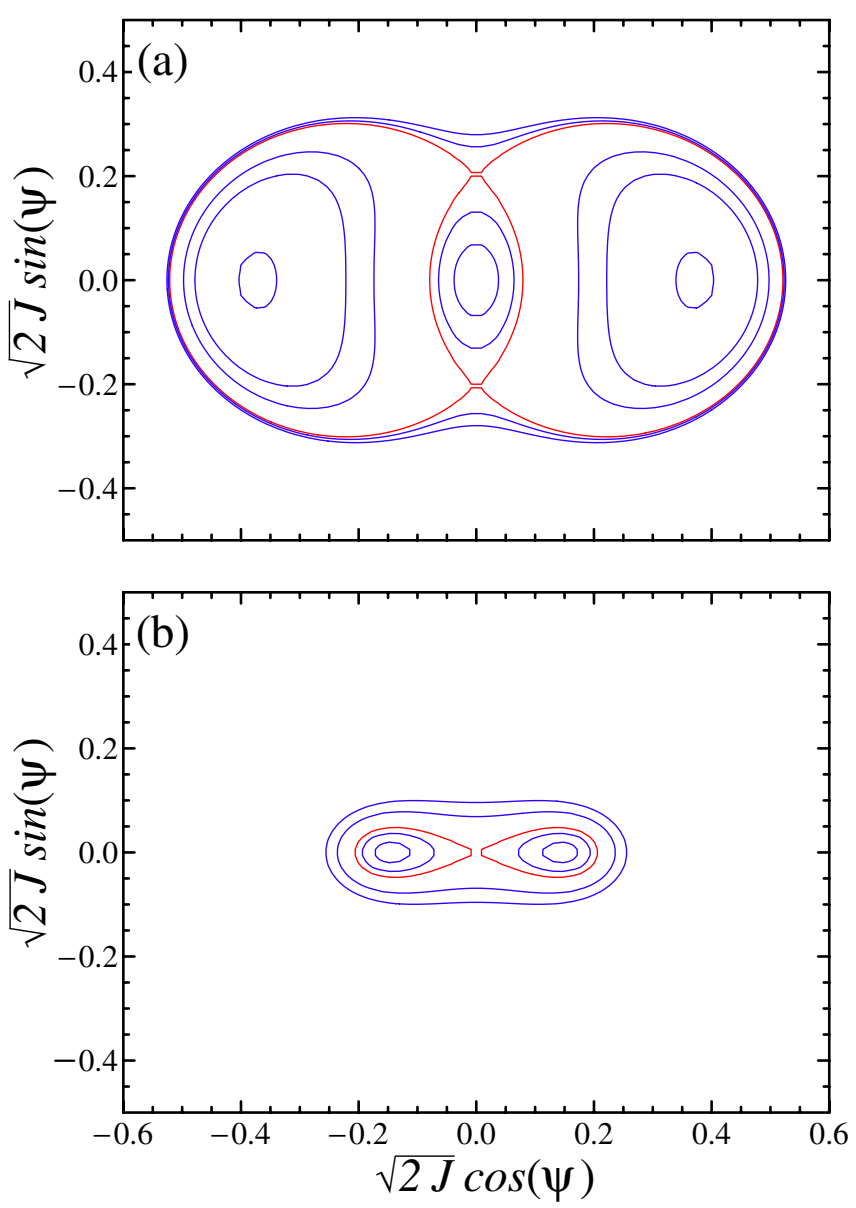

FIG. 4. (Color) Phase space of the modulated bunch with two different modulation frequencies in the rotating reference frame. (a) $\omega_{m} \leq(2-\epsilon / 2) \omega_{s}$ with 3 stable fixed points and (b) $\omega_{m}>$ $(2-\epsilon / 2) \omega_{s}$ with 2 stable fixed points. The red line corresponds to the separatrix. 


$$
J_{U F P}= \begin{cases}8\left(1-\frac{\omega_{m}}{2 \omega_{s}}\right)-2 \epsilon, & \omega_{m} \leq(2-\epsilon / 2) \omega_{s} \\ 0, & \omega_{m}>(2-\epsilon / 2) \omega_{s}\end{cases}
$$

corresponding to the angles $\psi_{U F P}=\pi / 2,3 \pi / 2$.

The phase-space coordinates of the new stable fixed points are related to the square root of $J_{S F P}$ or $J_{U F P}$ and are functions of the rf phase modulation amplitude and frequency.

The effect of phase modulation is to create new regions of stability inside the bunch, besides the original one. As the formation of those islands depends on the modulation amplitude and frequency, there is a continuum of forms from one island to the appearance of three islands when $\omega_{m} \leq(2-\epsilon / 2) \omega_{s}$ and the formation of only two islands when $\omega_{m}>(2-\epsilon / 2) \omega_{s}$. The longitudinal phase space for the situations described above is shown in Fig. 4.

\section{B. The effect of radiation}

All the arguments given above do not take into account the fact that electrons in a bunch lose energy through the emission of photons. This effect causes oscillations inside the bunch to damp, so it is important to know when this damping can destroy the formation of islands produced by phase modulation or modify some characteristics derived using the Hamiltonian formalism. The analysis that follows uses a simple nonlinear oscillation theory to calculate the trajectories of a single particle under the influence of phase modulation at the second harmonic of the synchrotron frequency.

The equation of motion for a particle subjected to phase modulation can be written as follows [19]:

$$
\frac{d^{2} \phi}{d t^{2}}+2 \gamma_{d} \frac{d \phi}{d t}+\omega_{s}^{2}\left[1+\epsilon \cos \left(\omega_{m} t\right)\right]=\beta \phi^{3}
$$

with $\gamma_{d}$ the radiation damping time and

$$
\epsilon=A_{m} \tan \bar{\phi}_{s} \quad \text { and } \quad \beta=\frac{1}{6} \omega_{s}^{2} .
$$

We also consider that $A_{m}$, the phase modulation amplitude, and $\phi$ are such that $A_{m} \ll \phi \ll 1 \mathrm{rad}$ and retain only the terms most important which are the parametric resonance term on the left side and the third order term on the right side of Eq. (10). Nonresonant terms in $A_{m}$ and $\tan \bar{\phi}_{s}$ are discarded.

From the standard analysis of parametric resonances [20], we expect that the solution of Eq. (10) has the form

$$
\phi(t)=a(t) \cos \left(\frac{\omega_{m}}{2} t\right)+b(t) \sin \left(\frac{\omega_{m}}{2} t\right) .
$$

Substituting the solution (12) in Eq. (10), we can write a set of equations for the functions $a$ and $b$ :

$$
\begin{aligned}
& 2 \dot{a}+2 \gamma_{d} a+(\alpha+\delta \omega) b+k b\left(a^{2}+b^{2}\right)=0, \\
& 2 \dot{b}+2 \gamma_{d} b+(\alpha-\delta \omega) b-k a\left(a^{2}+b^{2}\right)=0,
\end{aligned}
$$

where

$$
\alpha=\frac{\omega_{s} \epsilon}{2}, \quad k=\frac{\omega_{s}}{8}, \quad \delta \omega=\omega_{m}-2 \omega_{s} .
$$

If a particle is to be captured by the parametric resonance, the slowly varying coefficients $a(t)$ and $b(t)$ must increase exponentially. Taking only the lower terms in $a$ and $b$ in Eqs. (13) and (14) and using this condition we find that, in order for phase modulation to create stable islands in the longitudinal phase space, it is necessary that the condition $\alpha>2 \gamma_{d}$ be satisfied. Rewriting this condition in terms of the phase modulation amplitude, we find that ${ }^{1}$

$$
A_{m} \geq \frac{4 \gamma_{d}}{\left|\tan \phi_{s}\right| \omega_{s}} \approx 0.026 \mathrm{rad}
$$

and this is a limit imposed by radiation damping below which no island is observed.

Changing the longitudinal phase-space reference frame to one which rotates with a frequency of $\omega_{m} / 2$ in time, we find that the coordinates in this new frame are related only to the functions $a(t)$ and $b(t)$ and the fixed points are given by the solution of Eqs. (13) and (14) when $\dot{a}=\dot{b}=0$. Rewriting $a=\sqrt{2 r} \cos \theta$ and $b=\sqrt{2 r} \sin \theta$, we end up with the following solutions:

$$
\begin{gathered}
r=\frac{-\delta \omega \pm \sqrt{\alpha^{2}-4 \gamma_{d}^{2}}}{2 k}, \\
\tan \theta=\frac{-\alpha \pm \sqrt{\alpha^{2}-4 \gamma_{d}^{2}}}{2 \gamma_{d}} .
\end{gathered}
$$

If we set $\gamma_{d}=0$, we find that the stable fixed points are the ones already calculated in the previous section using the Hamiltonian formalism. To take into account the effect of radiation damping we define, using (17), an effective amplitude of modulation which is modified by the radiation damping effect as

$$
A_{m}^{\mathrm{eff}}=\sqrt{A_{m}^{2}-\left(\frac{4 \gamma_{d}}{\left|\tan \phi_{s}\right| \omega_{s}}\right)^{2}} .
$$

This quantity is particularly important when one uses the Hamiltonian formalism to make predictions about measurements. In these calculations $A_{m}^{\text {eff }}$ should be used instead of $A_{m}$ when the damping effects are not negligible.

\section{Dynamics of small oscillations around the fixed points}

We now consider the dynamics of particle oscillations in the vicinity of the stable fixed points (SFPs) in order to derive two important properties of the electron motion: the equilibrium density distribution close to the SFPs and the frequency of synchrotron oscillations around those points

\footnotetext{
${ }^{1}$ Calculated for a total accelerating voltage of $500 \mathrm{kV}$.
} 
(also known as the island tune), including its dependence on the oscillation amplitude. This is an important quantity since it describes the frequency of incoherent oscillation of the particles inside the island when subjected to a longitudinal kick and its dependence on oscillation amplitude is intimately related to the effectiveness of phase modulation as a damping mechanism for coherent synchrotron oscillations.

We start by expanding Eq. (6) around a fixed point with coordinates $\left(J_{0}, \psi_{0}\right)$ in the rotating reference frame [5]:

$$
\begin{aligned}
& \delta^{\prime}=-\sqrt{2 J} \sin \psi+\sqrt{2 J_{0}} \sin \psi_{0}, \\
& \phi^{\prime}=\sqrt{2 J} \cos \psi-\sqrt{2 J_{0}} \cos \psi_{0},
\end{aligned}
$$

and find that

$$
H^{\prime}=\frac{A}{2} \delta^{\prime 2}+\frac{B}{2} \phi^{\prime 2}+\mathcal{O}\left(\phi^{\prime 3}\right)
$$

where

$$
\begin{aligned}
A= & \omega_{s}-\frac{\omega_{m}}{2}-\frac{\omega_{s} J_{0}}{8}-\frac{\omega_{s} J_{0}}{4} \sin ^{2} \psi_{0} \\
& -\frac{\omega_{s} \epsilon}{4}\left(1-\frac{J_{0}}{6}+\frac{J_{0}}{4} \cos 2 \psi_{0}\right), \\
B= & \omega_{s}-\frac{\omega_{m}}{2}-\frac{\omega_{s} J_{0}}{8}-\frac{\omega_{s} J_{0}}{4} \cos ^{2} \psi_{0} \\
& +\frac{\omega_{s} \epsilon}{4}\left(1+\frac{J_{0}}{6}+\frac{J_{0}}{4} \cos 2 \psi_{0}\right) .
\end{aligned}
$$

In order to find the steady-state phase-space profile of the electron beam under phase modulation around a stable fixed point, we must solve the Fokker-Planck equation, using $H^{\prime}$ from Eq. (21) and taking into account the radiation damping $\left(\gamma_{d}\right)$ and quantum excitation $\left(\kappa=\sigma_{\delta}^{2} \gamma_{d}\right)$ terms. When all forces are balanced the solution of the Fokker-Planck equation is a bi-Gaussian where

$$
\Psi\left(\delta^{\prime}, \phi^{\prime}\right)=\frac{1}{2 \pi \sigma_{\delta} \sigma_{\phi}} \exp \left(-\frac{1}{2 \sigma_{\delta}^{2}} \delta^{\prime 2}-\frac{1}{2 \sigma_{\phi}^{2}} \phi^{\prime 2}\right)
$$

and the rms bunch energy and phase spread are

$$
\sigma_{\delta}=\sqrt{\frac{\kappa}{\gamma_{d}}} \quad \text { and } \quad \sigma_{\phi}=\sqrt{\frac{A}{B}} \sigma_{\delta}=\sqrt{\frac{A \kappa}{B \gamma_{d}}},
$$

where

$$
\gamma_{d}=\frac{1}{\tau_{\mathrm{rad}}} \approx 250 \mathrm{~s}^{-1} \text { and } \kappa \approx 4 \times 10^{-4} \mathrm{~s}^{-1} .
$$

In other words, particles close to the stable fixed points are subjected to an effective harmonic potential and we estimate the steady-state phase-space profile of the electron beam under phase modulation (when radiation excitation and damping are balanced) by a bi-Gaussian distribution.
If we go further in expanding (6) we find that

$$
\begin{aligned}
H^{\prime}= & \frac{A}{2} \delta^{\prime 2}+\frac{B}{2} \phi^{\prime 2}-\frac{\omega_{s} \sqrt{2 J_{S F P}}}{16} \phi^{\prime 3}-\frac{\omega_{s}}{16}\left(\frac{\phi^{\prime 4}+\delta^{\prime 4}}{4}\right) \\
& -\frac{\omega_{s} \epsilon}{24}\left(\frac{\phi^{\prime 4}-\delta^{\prime 4}}{4}\right)
\end{aligned}
$$

and that the unperturbed frequency, or island frequency, is given by $\omega=\sqrt{A B}$. This quantity is real for the stable fixed points and imaginary for the unstable ones.

Changing variables to

$$
\phi^{\prime}=\sqrt{2 J^{\prime}} \sqrt{\frac{A}{B}} \cos \Psi^{\prime} \quad \text { and } \quad \delta^{\prime}=-\sqrt{2 J^{\prime}} \sqrt{\frac{B}{A}} \sin \Psi^{\prime}
$$

and using the canonical perturbation technique [21] with the generating function

$$
F_{2}\left(\Psi^{\prime}, I\right)=\Psi^{\prime} I+G_{3}(I) \sin 3 \Psi^{\prime}+G_{1}(I) \sin \Psi^{\prime},
$$

where the functions $G_{1}(I)$ and $G_{3}(I)$ are chosen so that the term in $J^{3 / 2}$ is canceled, we find that the phase averaged Hamiltonian is

$$
\begin{aligned}
\langle H\rangle_{\Psi}(I) \approx & \omega I-\frac{\omega_{s}}{16} I^{2}\left[\frac{3}{8} \frac{A^{2}+B^{2}}{A^{2} B^{2}}+\frac{\epsilon}{4} \frac{A^{2}-B^{2}}{A^{2} B^{2}}\right] \\
& -\frac{15}{16} \frac{\omega_{s}^{2} \sqrt{I_{S F P}}}{\omega}\left(\frac{A}{B}\right)^{3 / 2} I^{2}
\end{aligned}
$$

and the revolution frequency around each stable fixed point for a particle with a phase amplitude $\hat{\phi}$ with respect to that point is

$$
\omega(\hat{\phi}) \approx \omega\left(1-\frac{3 \omega_{s}}{16} \frac{A^{2}+B^{2}}{A^{2}|B|} \frac{\hat{\phi}}{8}\right)
$$

where the parameters of the rf phase modulation are in the coefficients $A$ and $B$.

\section{Beam transfer function and Landau damping}

To explain why phase modulation has such an intense effect on the amplitude of CBM oscillations, we consider that phase modulation creates a spread in the frequency distribution inside the bunch thus increasing the amount of Landau damping [22]. In this case we are only interested in the damping of synchrotron oscillations of the bunch centroid which are produced by the coupling between the synchrotron motion and a longitudinal HOM of the $\mathrm{rf}$ cavity. We therefore proceed to calculate the response of the beam centroid motion to an external harmonic perturbation when phase modulation is on.

Suppose the particles in a bunch have a distribution $\Psi(r)$ in phase space. In case the beam is phase modulated the distribution can be approximated, in the rotating reference frame, by three Gaussian functions each one centered at a fixed point 


$$
\begin{aligned}
\Psi_{0}(r)= & \frac{N_{c}}{\sqrt{2 \pi} \sigma_{c}} e^{-r^{2} / 2 \sigma_{c}^{2}}+\frac{N_{i 1}}{\sqrt{2 \pi} \sigma_{i}} e^{-\left(r-r_{i 1}\right)^{2} / 2 \sigma_{i}^{2}} \\
& +\frac{N_{i 2}}{\sqrt{2 \pi} \sigma_{i}} e^{-\left(r-r_{i 2}\right)^{2} / 2 \sigma_{i}^{2}},
\end{aligned}
$$

where the indices $c$ and $i 1,2$ indicate the central island or the side islands formed when the phase modulation is turned on, $r$ is a radial coordinate in the longitudinal phase space, and $N_{j}$ is the relative number of particles in each island such that $N_{i 1}+N_{i 2}+N_{c}=1$.

Considering that, when an external perturbation with amplitude $F_{0}$ acts on the beam, a dipolar motion of the centroid is excited, we can write the electron distribution as

$$
\Psi(r, \theta, t)=\Psi_{0}(r)+\Psi_{1}(r) e^{-j(\Omega t-\theta)},
$$

where $\Psi_{1}$ is a small perturbation. Using (33) and considering that the equation of motion for each particle inside the island and near the SFPs can be approximated by

$$
\ddot{\tau}+2 \gamma_{d} \dot{\tau}+\nu_{c, i}^{2} \tau=F_{0} e^{-j \Omega t},
$$

where $\tau$ is the relative time displacement and $\nu_{c, 1}$ the island frequency, we can show that [23]

$$
\begin{aligned}
\Psi_{1}(r)= & \frac{N_{c} F_{0}}{\omega_{c}\left(\Omega-\omega_{c}\right)} \frac{\partial \Psi_{0 c}}{\partial r}+\frac{N_{i 1} F_{0}}{\omega_{i}\left(\Omega-\omega_{i 2}\right)} \frac{\partial \Psi_{0 i 1}}{\partial r} \\
& +\frac{N_{i 2} F_{0}}{\omega_{c}\left(\Omega-\omega_{i 2}\right)} \frac{\partial \Psi_{0 i 2}}{\partial r},
\end{aligned}
$$

in which we introduced the damping rate due to radiation defining a complex frequency for each island: $\omega_{c, i}=$ $\nu_{c, i}-j \gamma_{d}$.

The centroid motion is given by the first moment of the phase-space distribution

$$
\begin{aligned}
\bar{\tau}(\Omega) & =\int_{0}^{2 \pi} \int_{0}^{\infty} r^{2} \cos \theta d \theta d r \Psi(r, \theta, t) \\
& =\frac{F_{0}}{2 \omega_{c}}\left[N_{c} I_{c}(\Omega)+N_{i} \frac{\omega_{c}}{\omega_{i}} I_{i}(\Omega)\right] e^{-j \Omega t},
\end{aligned}
$$

where the contribution of the side islands results in the same integral since their frequencies are the same.

The function $I_{n}(\Omega)$ is the dispersion integral and is defined as

$$
I_{n}(\Omega) \equiv \pi \int_{0}^{\infty} \frac{r^{2} d r}{\Omega-\omega_{n}(r)} \frac{\partial \Psi_{0}}{\partial r}
$$

and the BTF for a beam under phase modulation in the rotating reference frame is

$$
I(\Omega)=N_{c} I_{c}(\Omega)+N_{i} \frac{\omega_{c}}{\omega_{i}} I_{i}(\Omega) .
$$

As the measurements are performed in the laboratory reference frame it is necessary to transpose Eq. (38) to this reference frame. To do this, note that in the lab frame the frequency of the lateral islands appears as a lower sideband of half of the modulation frequency [Appendix B] so that we have two different frequency responses: $\omega_{m} / 2+\omega_{c}$ for the central island and $\omega_{m} / 2-$ $\omega_{i}$ for the side islands, as shown in Fig. 5. It is interesting to observe that the effect of phase modulation is to create a second peak, related to the island frequency and also an intermediary phase jump in the BTF; these features are very different from the conventional picture expected when measuring the BTF of an electron beam without phase modulation.

It is possible to estimate the effect of phase modulation on the damping time of the centroid motion if we calculate the increase in frequency spread due to modulation. The particle distribution in the frequency domain is given by

$$
\begin{aligned}
\Psi_{0}^{\prime}(\omega)= & \frac{N_{c}}{\Delta \omega_{c}} e^{-\left(\omega_{c}-\omega\right) / \Delta \omega_{c}} H\left(\omega_{c}-\omega\right) \\
& +\frac{N_{i}}{\Delta \omega_{i}} e^{-\left(\omega_{i}-\omega\right) / \Delta \omega_{i}} H\left(\omega_{i}-\omega\right),
\end{aligned}
$$

and shown in Fig. 6, where $H(x)$ is a step function. Defining the frequency spread as

$$
\Delta \omega=\sqrt{\left\langle\omega^{2}\right\rangle}=\left[\int \omega^{2} \Psi_{0}^{\prime}(\omega) d \omega\right]^{1 / 2},
$$

we have that the ratio between the natural damping time to the one created when the beam is under phase modulation is
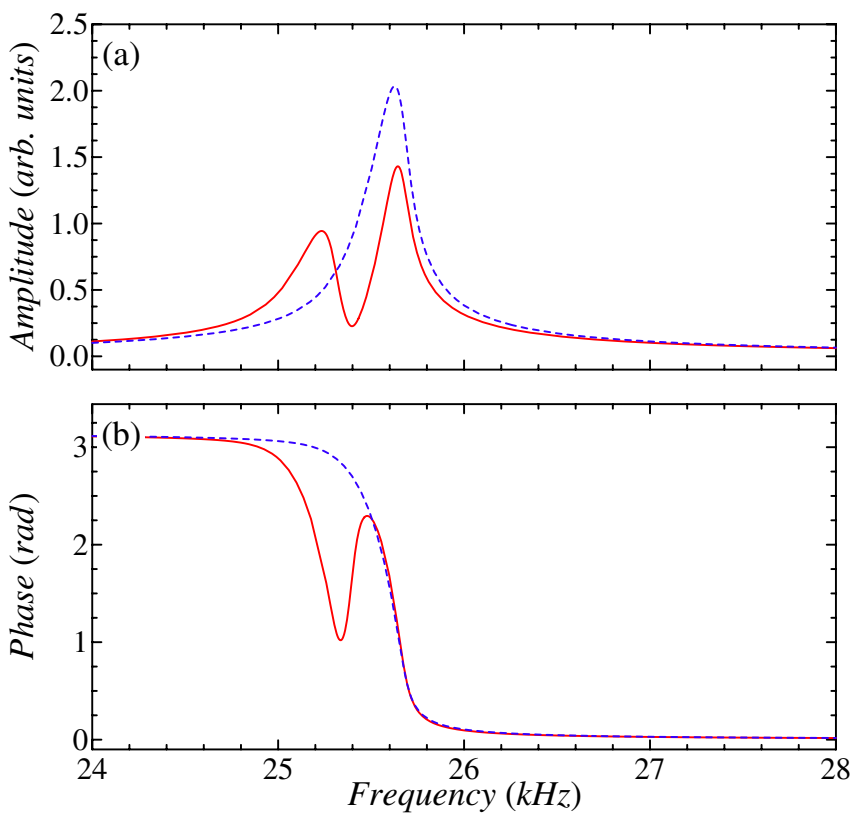

FIG. 5. (Color) Calculated amplitude and phase of the beam transfer function for a bunch with (full red line) and without (dashed blue line) rf phase modulation and with half of the particles in the central stable island. 


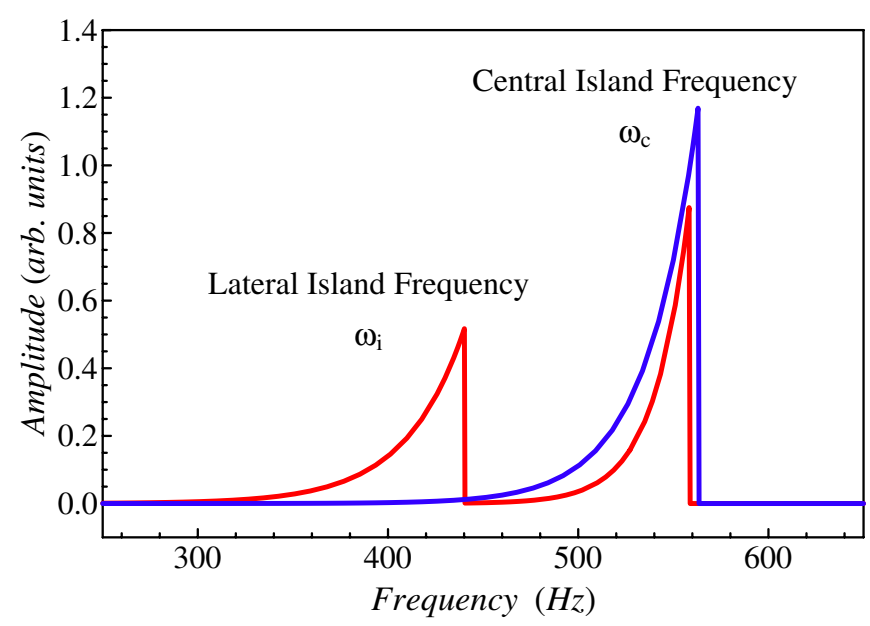

FIG. 6. (Color) Calculated distribution of incoherent frequencies in the rotating reference frame (blue curve) without phase modulation and (red curve) with phase modulation turned on, $f_{m}=50 \mathrm{kHz}, A_{m}=50 \mathrm{mrad}$ and half of the particles in the central island.

$$
\frac{\tau_{\mathrm{mod}}}{\tau_{\text {nat }}}=\frac{\Delta \omega_{\mathrm{nat}} / 2 \pi+1 / \tau_{\mathrm{rad}}}{\Delta \omega_{\mathrm{mod}} / 2 \pi+1 / \tau_{\mathrm{rad}}} .
$$

The natural spread in the incoherent frequency for the bunch in the LNLS electron storage ring is $\Delta \omega_{\text {nat }} \approx$ $160 \mathrm{~Hz}$ and the total damping, including the radiation damping time, related to this frequency dispersion is $\tau_{\text {nat }} \approx$ $3 \mathrm{~ms}$. Suppose half of the particles are in the central island, so that $N_{c}=1 / 2$, and the amplitude and frequency of the modulation are $50 \mathrm{mrad}$ and $50 \mathrm{kHz}$, respectively. The frequency spread and the new damping time due to phase modulation, given by Eqs. (40) and (41), are $\Delta \omega_{\bmod }=$ $490 \mathrm{~Hz}$ and $\tau_{\text {mod }}=2.2 \mathrm{~ms}$. This damping time is smaller than the estimated growth time for the L1 instability, which is $3.3 \mathrm{~ms}^{2}$ So, using the phase modulation we are able to set the beam well below the stability boundary and effectively damp this coupled-bunch mode.

So far we have only estimated the effects of phase modulation on beam stability. We are going now to calculate the implications of the increase in frequency spread due to modulation on the beam when it is subjected to its own fields.

The spread in incoherent frequencies in the bunch causes it to strongly damp coherent oscillations excited by a harmonic driving force. This force can be an external excitation or wakefield which interacts with the electron beam [22]. If we consider a short bunch which interacts with an impedance of the machine, such as a HOM, the centroid motion is governed by the following equation:

\footnotetext{
${ }^{2}$ This value for the mode growth time is for a temperature of $40^{\circ} \mathrm{C}$, an accelerating voltage of $500 \mathrm{kV}$, and a total stored current of $250 \mathrm{~mA}$.
}

$$
\frac{d^{2} \tau}{d s^{2}}+\frac{\omega_{0}^{2}}{v^{2}} \tau=-\frac{j e^{2} N \eta \omega_{\mathrm{rev}} \bar{\tau}}{\beta^{2} E_{0} C^{2}} Z_{\|}
$$

where $s=v t$ with $v=\beta c$ is the particle velocity, $N$ is the number of electrons in the bunch, $e$ is electron charge, $\omega_{\text {rev }}$ is the revolution frequency, $E_{0}$ is the particles energy, $C$ is the orbit length, and $Z_{\|}$is the effective longitudinal impedance [22].

Introducing the ansatz

$$
\bar{\tau}(s)=B e^{-j \Omega s / v},
$$

the coherent synchrotron tune shift can be defined as

$$
\left(\Delta \omega_{s}\right)_{\mathrm{coh}}=\Omega-\omega_{0}=-\frac{j e^{2} N \eta \omega_{\mathrm{rev}} c^{2}}{2 \omega_{0} E_{0} C^{2}} Z_{\|},
$$

and the stability condition is so that: $\operatorname{Im}(\Omega)<0$.

Therefore, using Eqs. (36) and (44) we can define the stability diagram for coherent centroid oscillations in the following way:

$$
U+i V=-j\left(\Delta \omega_{s}\right)_{\mathrm{coh}}=\frac{j}{I(\Omega)}=\frac{\hat{g}(\Omega)+j \hat{f}(\Omega)}{\hat{g}^{2}(\Omega)+\hat{f}^{2}(\Omega)},
$$

where $\hat{f}(\omega)$ and $\hat{g}(\omega)$ are the real and imaginary parts of the BTF $I(\Omega)$ [Eq. (37)].

Figure 7 shows the stability diagram for a bunch under phase modulation (black full line) and in normal conditions (dashed line). Note that, when phase modulation is turned on, the stable area is increased and that a new region, inside the loop, is formed. This new region is also unstable as shown by the red line, which is calculated for a positive imaginary coherent shift, that violates the stability criterion. The dots shown in Fig. 7 represent various operation points depending on the operating temperature of the new

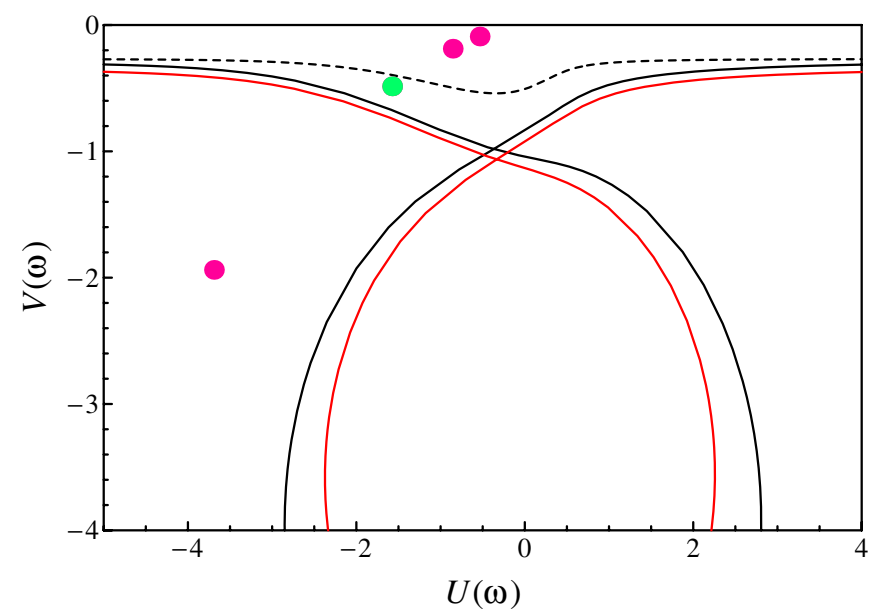

FIG. 7. (Color) Stability diagram in the $U \times V$ plane (black full line) phase modulation on and for $50 \%$ of electrons in the innermost stable island, (red full line) unstable line for a positive imaginary coherent frequency shift, (dashed line) phase modulation off, and (dots) various operating points for temperatures of the new cavity varying from $40{ }^{\circ} \mathrm{C}$ to $55^{\circ} \mathrm{C}$. 
cavity. The green mark, for example, indicates the point for $50^{\circ} \mathrm{C}$ that is unstable under normal operation conditions and becomes stable if phase modulation is turned on.

\section{SIMULATION}

In order to gain some insight into the behavior of the bunches under phase modulation and verify the properties derived from the theoretical model outlined above, we developed a longitudinal dynamics simulation code including beam loading effects and the presence of the longitudinal HOMs as well as phase modulation. The tracking code calculates the trajectories of individual particles in longitudinal phase space and derives the corresponding averaged quantities representing the motion of the bunch centroid as well as a projected (time-averaged) longitudinal charge density profile appropriate for comparison with experimental results.

Even though the application of the code to the single bunch case is relatively straightforward, the calculations for the multibunch case are much more time consuming, since at the LNLS storage ring we routinely operate with all 148 bunches filled (no gaps in the bunch train). In order to circumvent that difficulty, we use a hybrid simulation, in which we treat the first 147 bunches $\left(b_{1}-b_{147}\right)$ as macroparticles (no internal structure) whereas the last bunch $\left(b_{0}\right)$ is composed of 1000 individual macroparticles. Note that although each one of the 147 bunches without internal structure is represented basically by a single point in phase space (rather than by a distribution), an effective bunch length (representing the projection of the phase-space bunch distribution on the time axis) is also attributed to those bunches when calculating the excitation of the wakefields. In fact, as the particles inside $b_{0}$ are redistributed in phase space as a result of phase modulation and interactions with the environment, its bunch length is also changed and that value of bunch length is then transferred to all nonstructured bunches $\left(b_{1}-b_{147}\right)$. In this way, one important aspect of the internal bunch structure is shared by all bunches. Clearly, this procedure cannot take into account all properties related to the internal motion of the bunches and describe their effect on the stabilization of the motion. However, we shall see in the following sections that such simulations give additional indication that the increase in incoherent frequency spread inside the bunches is indeed the main stabilizing mechanism provided by phase modulation.

\section{A. Equations of motion}

The model used in the simulation considers that every possible bunch is filled with electrons and each one of those bunches, except one, is represented by a single macroparticle. The bunches pass periodically through the accelerating cavities and contribute to the cumulative buildup of the induced fields. The effect of the beam loading is also taken into account in the calculation of the trajectories of the individual electrons of the structured bunch.

In order to calculate the trajectories of these macroparticles in the longitudinal phase space, we use the following equations:

$$
\begin{gathered}
\tau_{b, n}=\tau_{b, n-1}-\alpha \delta_{b, n-1} T_{0}, \\
\delta_{b, n}=\left(1-2 \gamma_{d} T_{0}\right) \delta_{b, n-1}+\frac{\left(e V_{\mathrm{tot}}-U_{0}\right)}{E},
\end{gathered}
$$

with

$$
V_{\mathrm{tot}}=V_{\mathrm{rf}}+V_{b l},
$$

where $\tau$ and $\delta$ are the time displacement and energy deviation of the macroparticle related to the reference synchronous particle, $b$ is the index of the bunch and $n$ is the number of turns, $T_{0}$ is the revolution period, $V_{\mathrm{rf}}$ is the gap voltage, and $V_{b l}$ is the energy drained by the beam loading effect, which takes into account the beam loading of the fundamental mode and of the longitudinal mode (L1). In order to simulate each particle in the structured bunch $\left(b_{0}\right)$, we use the same equations and also consider radiation excitation.

The phase modulation is included in the calculation of the rf voltage as follows:

$$
V_{\mathrm{rf}}=V_{0} \sin \left[\phi_{s}-\omega_{\mathrm{rf}} \tau_{b, n}+A_{m} \sin \left(\omega_{m} t\right)\right],
$$

where $V_{b l}$ is the peak voltage, $\phi_{s}$ the synchronous phase, $\omega_{\mathrm{rf}}$ the angular rf frequency, $A_{m}$ the modulation amplitude, and $\omega_{m}$ the modulation frequency which we set near the synchrotron second harmonic $\left(\omega_{m}=2 \omega_{s}+\delta \omega\right)$.

The beam loading effect is introduced in the tracking simulation using the following expressions:

$$
V_{n}=V_{n-1} e^{-\omega_{\mathrm{res}} \Delta t / 2 Q_{L}+j \omega_{\mathrm{res}} \Delta t}-k q_{0} e^{-\omega_{\mathrm{res}}^{2} \sigma_{\tau}^{2}},
$$

where $\omega_{\text {res }}$ is the angular frequency, $k$ is the loss factor of the mode, $q_{0}$ is the charge per bunch, and $\sigma_{\tau}$ is the rms bunch length.

\section{B. Single bunch simulations}

Using the simulation code described above, we calculate the phase-space distribution of the particles in a single bunch of 1000 macroparticles under phase modulation. Figure 8 shows the phase space with two different modulation frequencies and also without phase modulation. The phase space patterns observed are in good agreement with the ones calculated using the Hamiltonian (6) and shown in Fig. 4. In particular, the position of the islands center given by simulation corresponds to the action amplitudes $J_{S F P}=$ 0.122 and 0.047 for 3 and 2 islands, respectively, as shown in Fig. 8. This is in very good agreement with the theoretical values 0.130 and 0.042 for the cases with 3 and 2 islands, obtained from (8) using the effective modulation amplitude $\left(A_{m}^{\text {eff }}\right)$. 


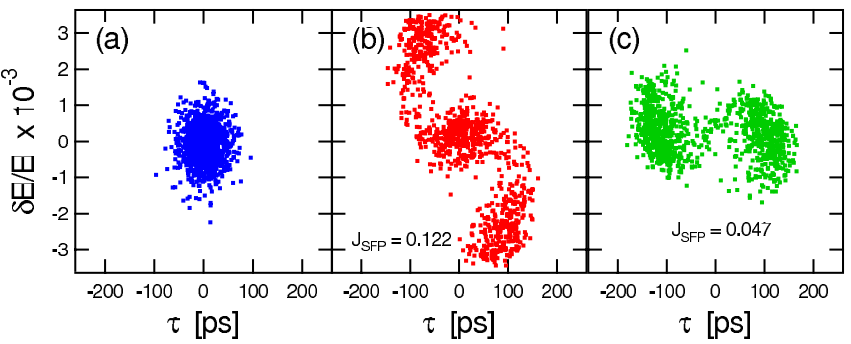

FIG. 8. (Color) Single bunch (1000 macroparticles) simulation showing the phase space for three different situations (a) without modulation, (b) with $f_{m}<(2-\epsilon / 2) f_{s}$, and (c) $f_{m}>(2-$ $\epsilon / 2) f_{s}$. In the two last figures it is possible to observe the formation of three and two islands, respectively, as predicted by the theory.

We also calculate the time-averaged longitudinal particle distribution profiles in time domain, which is a histogram of the particles position in time for a period much longer than the synchrotron one. This result simulates the profile that would be seen when measuring the signal from a button pickup, and is shown in Fig. 9.

Finally, we simulate the damping of a coherent dipolar oscillation by phase modulation. The dipolar oscillation of a high current single bunch $(30 \mathrm{~mA})$ is excited by the L1 HOM and, when phase modulation is turned on, the oscillation amplitude is reduced by a factor 30 (Fig. 10), which corresponds approximately to an attenuation of $30 \mathrm{~dB}$. This is similar to the $40 \mathrm{~dB}$ damping of the dipolar spectral line produced by phase modulation (Fig. 3) that we typically observe in multibunch mode. Note that in this single bunch simulation the effects of an increased incoherent frequency spread are fully taken into account.

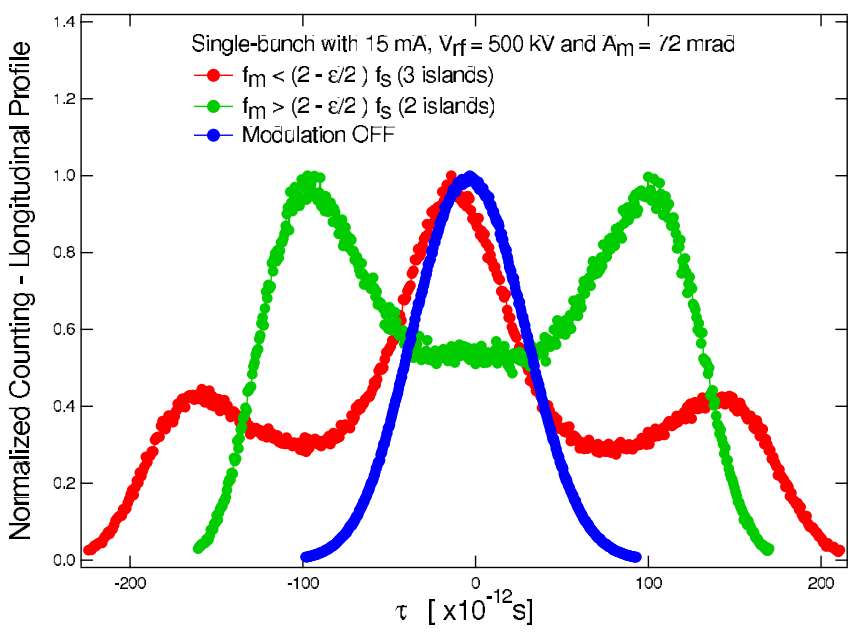

FIG. 9. (Color) Longitudinal profile in time domain of a simulation of 1000 macroparticles in three different situations: (blue) without modulation, (red) with $f_{m}<(2-\epsilon / 2) f_{s}$, and (green) $f_{m}>(2-\epsilon / 2) f_{s}$. Those patterns simulate the measurements of the signal of a button pickup.

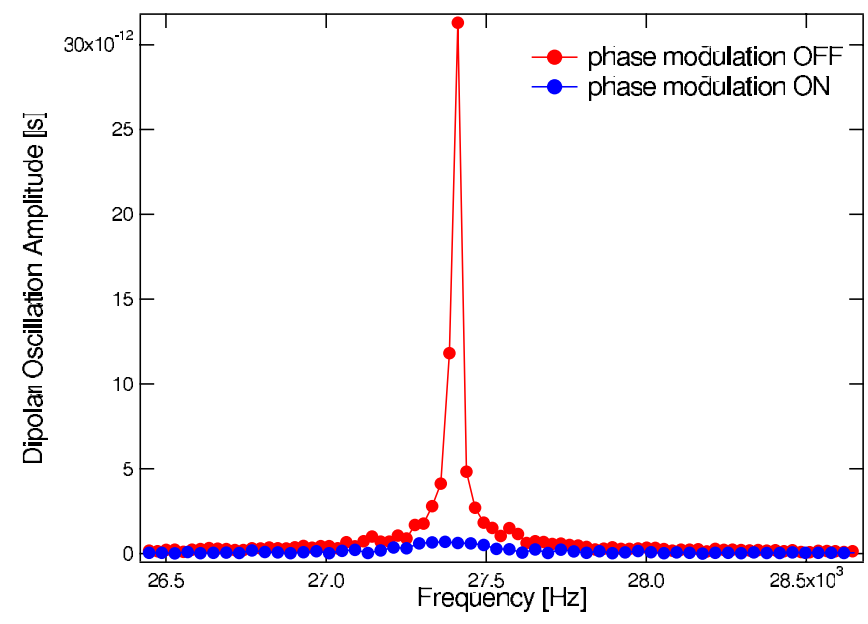

FIG. 10. (Color) Amplitude of the dipolar oscillation of the bunch centroid excited by a rf cavity longitudinal HOM for a single bunch with $30 \mathrm{~mA}$.

\section{Multibunch simulations}

For the multibunch case, we simulate the dipolar oscillation excited by the longitudinal HOM of the cavities [Fig. 11(a)]. The simulations are performed considering a total stored current of $200 \mathrm{~mA}$. A comparison between two different multibunch tracking simulations shows that the change in the amplitude of the dipolar coherent synchrotron oscillation when the L1 mode is turned on is greater than $100 \mathrm{~dB}$, demonstrating the excitation of dipolar oscillations in the beam, as observed during user shifts. We could also observe a good agreement between the value of the growth time of the L1 mode given by theory $(4 \mathrm{~ms})$ and simulation $(4.5 \pm 0.5 \mathrm{~ms})$.

Figures 11(a) and 11(b) show results of multibunch simulations without phase modulation for two different equilibrium bunch lengths. We see that, even if the bunch length in increased by a factor of 3 (which is larger than the increase observed in multibunch simulations when phase modulation is applied), there is basically no damping of the coherent oscillations excited by the L1 HOM. In other words, the mere dilution of the beam bunches is not enough to explain the coherent damping efficiency of phase modulation: the internal rearrangement of longitudinal phase space and corresponding increased frequency spread is a fundamental ingredient, as could be seen in the single bunch simulations above.

Finally, we simulate a multibunch case with phase modulation, which is shown in Fig. 11(c). Now we observe a damping of the CBM amplitude by a factor 5, which is much smaller than what is observed in the single bunch simulations as well as in experiments. This is a result of the hybrid character of our simulation, in which only one out of 148 bunches has an internal structure (and corresponding frequency spread), although all bunches share the common increased bunch length. The damping of the 

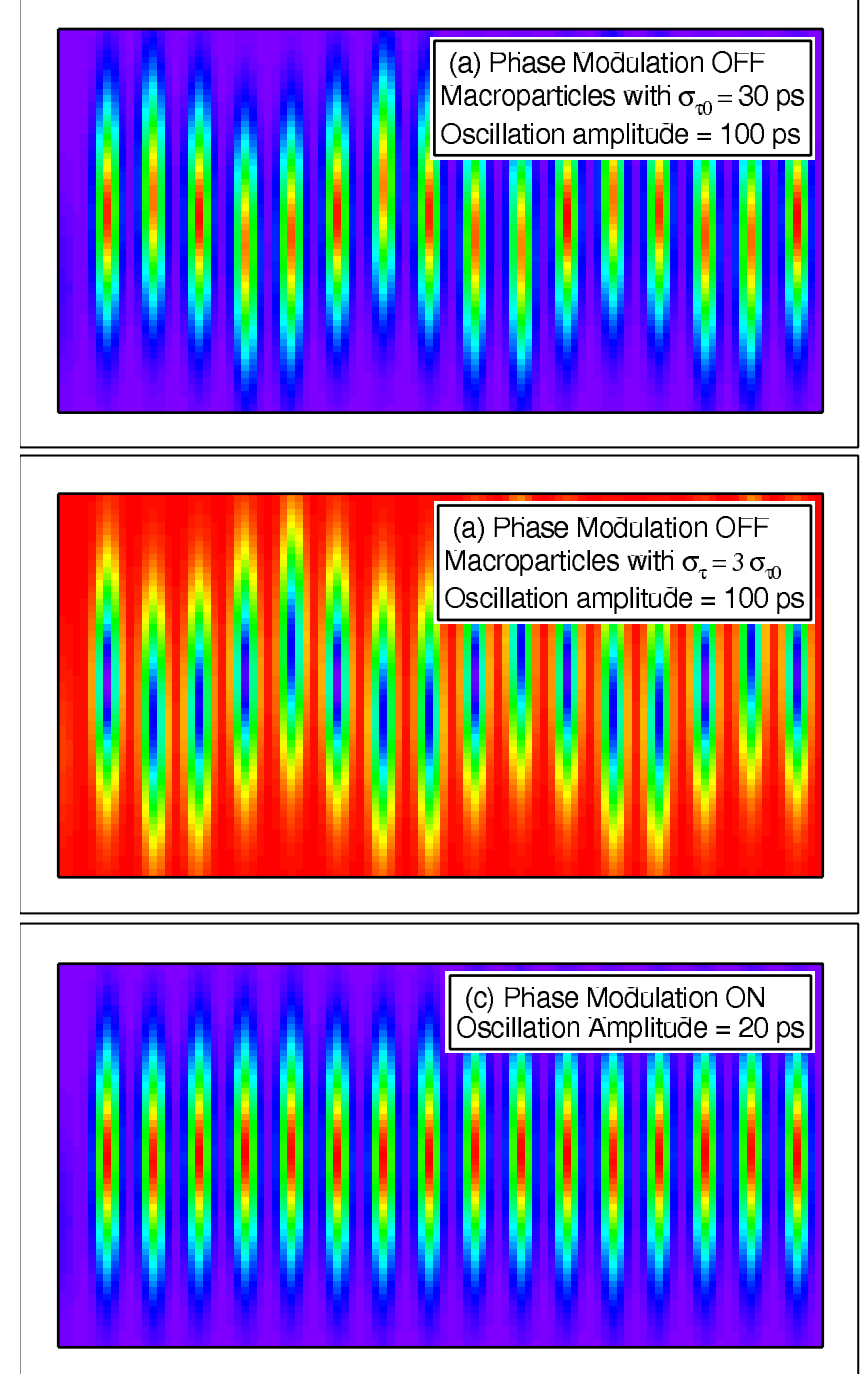

FIG. 11. (Color) Simulation of the centroid oscillation of a longitudinal CBM driven by an rf cavity HOM: (a) without phase modulation, (b) without phase modulation and 3 times the natural bunch length, and (c) with phase modulation. The figure shows one of each 6 bunches, as it would be seen by a streak camera.

structured bunch $b_{0}$ weakens the coupling between the electron beam and the HOM reducing the amplitude of the CBM coherent oscillation.

\section{MEASUREMENTS AND RESULTS}

In Secs. III and IV we calculate and simulate some characteristics of the longitudinal beam dynamics with $\mathrm{rf}$ phase modulation on the second harmonic of the synchrotron frequency. In this section we perform an experimental verification of the predictions made. The details of the experimental setup and the results obtained are shown in the following parts.

\section{A. Experimental setup}

The basic idea of the experiments is to excite the beam via phase modulation of the rf fields and observe its response as a function of a controlled parameter such as modulation amplitude and frequency. To modulate the phase of the accelerating fields, we use a voltage driven phase shifter attached to the output of the rf generator responsible for producing the master if signal. This phase shifter is controlled by a signal from another generator that creates a modulation with a chosen frequency. The full experimental setup is shown in Fig. 12.

The shape of the bunch is measured by a wideband oscilloscope (HP 54750A, $20 \mathrm{GHz}$ ) and the amplitude of the dipolar synchrotron sideband is observed using a spectrum analyzer (HP8560) both receiving the signal from a button pickup in the ring. In the multibunch case we choose to observe the synchrotron line around harmonic No. 281 of the revolution frequency that is close to the frequency of the L1 longitudinal HOM from the cavity.

We also measure the damping time for synchrotron oscillation of the centroid of a single bunch by comparing the phase between the master rf signal to the $476.066 \mathrm{MHz}$ component of the longitudinal beam signal and observe the signal in a high sampling rate oscilloscope (TDS684B). To create a longitudinal kick, we use a function generator that produces a step function with a given amplitude and acts on a phase shifter in series with the one used to modulate the rf wave. The additional setup required to measure the damping time is shown by the dashed lines in Fig. 12.

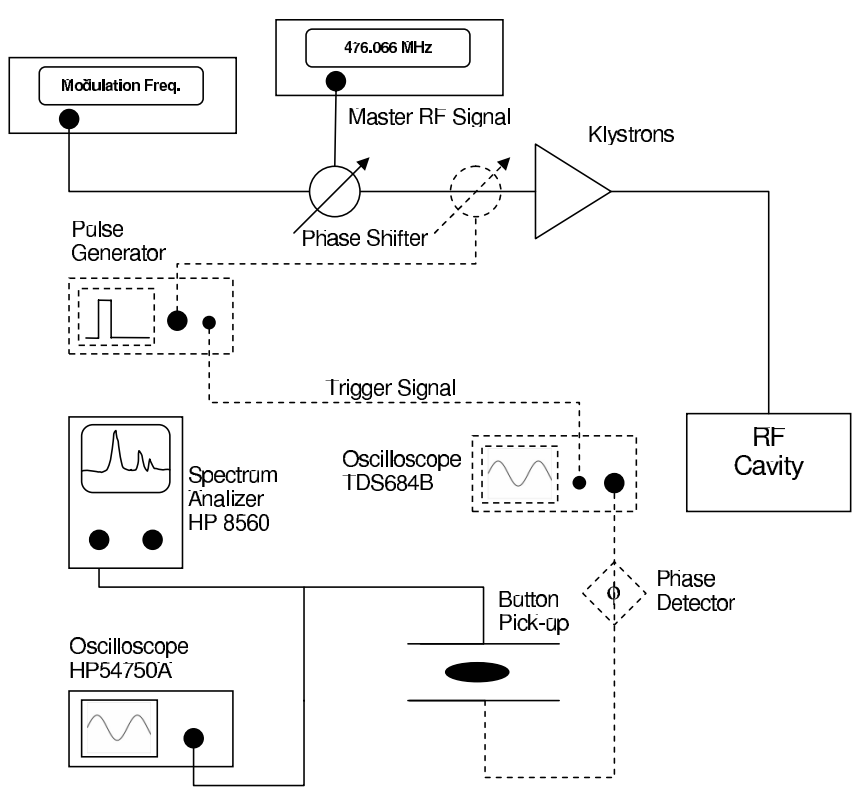

FIG. 12. General layout of the experimental setup. The solid line corresponds to the experimental setup used to measure the bunch shape and the beam spectrum while the dashed line corresponds to the additional setup used to measure the damping time for coherent synchrotron oscillations. 


\section{B. Experimental results and comparison with theory}

In this section we present a comparison between the predictions of the theoretical model and measurements. This analysis is done in the following order: island formation as a function of modulation frequency, damping time of the centroid synchrotron motion as a function of modulation frequency and amplitude, measurement of island tune and its behavior as a function of modulation amplitude and frequency, and measurement of the BTF.

\section{Island formation}

The time-averaged longitudinal bunch profile predicted by our single bunch simulation, Fig. 9 , is compared with the experimental results of the signal from a button pickup observed on an oscilloscope and shown in Fig. 13. We observe that, for modulation frequencies $\omega_{m}<\omega_{s}(2-$ $\epsilon / 2)$, the profile corresponds to the case with three stable regions whereas, when $\omega_{m}>\omega_{s}(2-\epsilon / 2)$, only two stable regions are created. This is an indirect way to observe the bunch internal structure created by phase modulation. The same result has already been obtained directly in other laboratories [11] using a streak camera to observe the beamlets.

\section{Longitudinal damping time}

The measurements of the damping of coherent synchrotron oscillations are performed using a function generator and a phase shifter to apply a longitudinal kick to the beam. Using the signal from a button pickup, we measure the phase of the rf component of the beam with respect to the master rf signal. Figure 14 is an example of the measurement for a single bunch with $4.3 \mathrm{~mA}$ and Fig. 15 is the

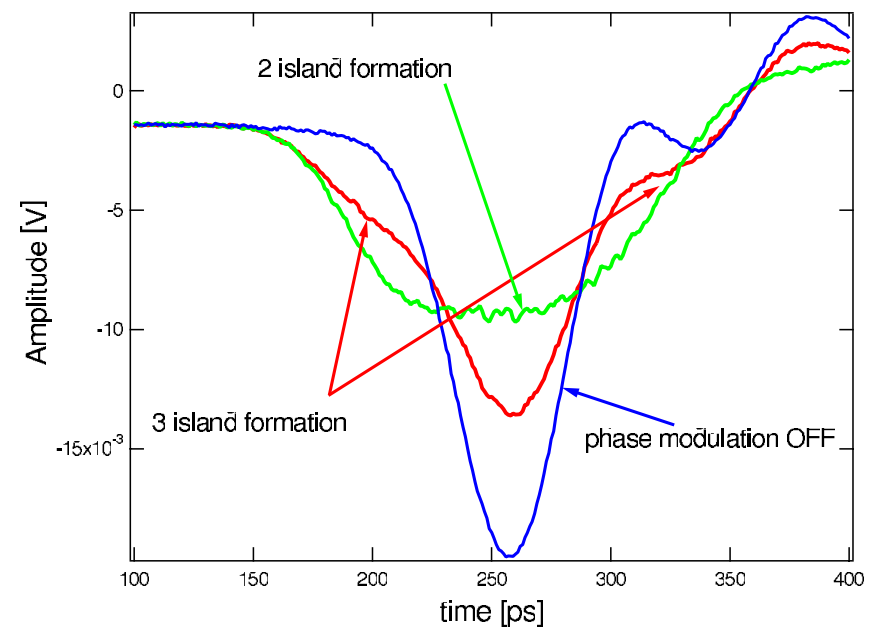

FIG. 13. (Color) Beam profile of a single bunch with $5 \mathrm{~mA}$ observed in an oscilloscope for a gap voltage of $460 \mathrm{kV}$. (blue curve) rf phase modulation off; (red curve) $A_{m}=60 \mathrm{mrad}$ and $f_{m}=f_{s}-0.2 \mathrm{kHz}$, and (green curve) $A_{m}=60 \mathrm{mrad}$ and $f_{m}=f_{s}+0.2 \mathrm{kHz}$.

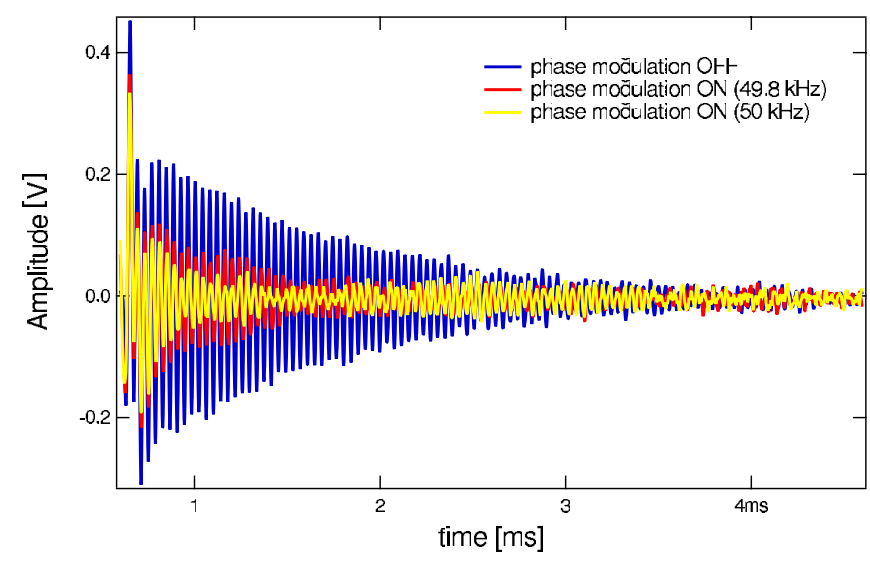

FIG. 14. (Color) Example of a measurement of the synchrotron damping time.

damping time ratio for a single bunch with phase modulation turned on and off.

The results presented in Fig. 14 are not the ones observed directly on the oscilloscope. When performing this measurement, we observe that the phase of the rf component of the beam oscillates not only with the synchrotron frequency but also with multiples and submultiples of the modulation frequency. The raw data acquired is filtered and those frequencies removed from the spectrum in order to leave just a band of frequencies around the dipolar frequency of centroid oscillations, which allows us to determine the damping time of coherent dipolar oscillations.

A set of measurements of damping time versus modulation frequency is shown in Fig. 15 and it is possible to observe that phase modulation is effective only for a narrow band of frequencies approximately $400 \mathrm{~Hz}$ wide. For frequencies above $50.2 \mathrm{kHz}$ the effect no longer exists, which agrees with the theoretical model used to describe the damping mechanism. This model predicts that, for

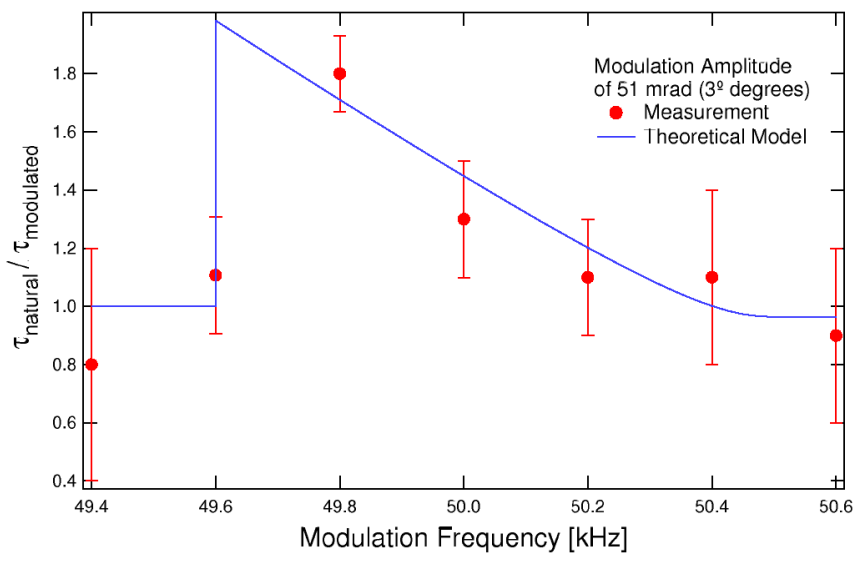

FIG. 15. (Color) Increase in damping time as a function of modulation frequency for a gap voltage of $460 \mathrm{kV}$ : (red dots) measurements and (blue curve) theoretical predictions calculated for an island population of $50 \%$. 
frequencies above $50.3 \mathrm{kHz}\left[f_{m}=(2+\epsilon / 2) f_{s}\right]$, the islands collapse into one so that there is no increase in damping time.

Using Eq. (41), we can estimate the value for the damping time increase due to phase modulation and Fig. 15

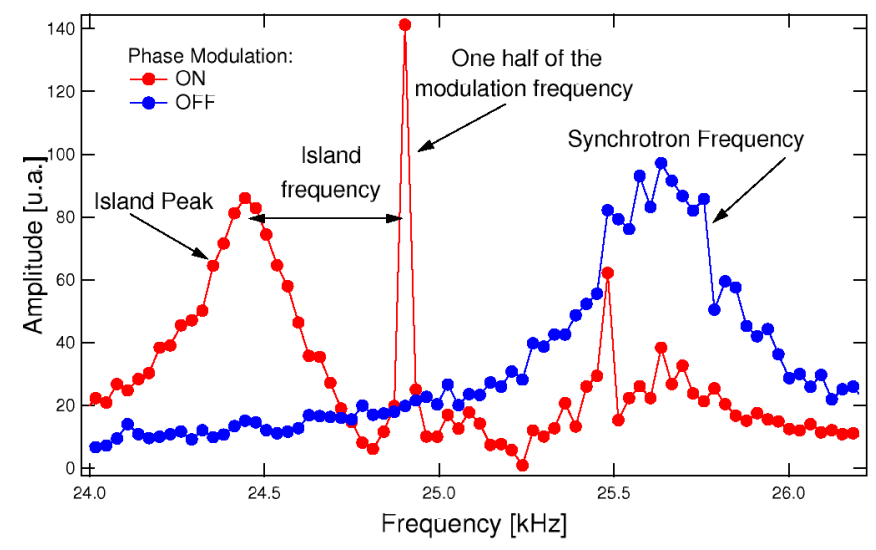

FIG. 16. (Color) Observation of the island tune.

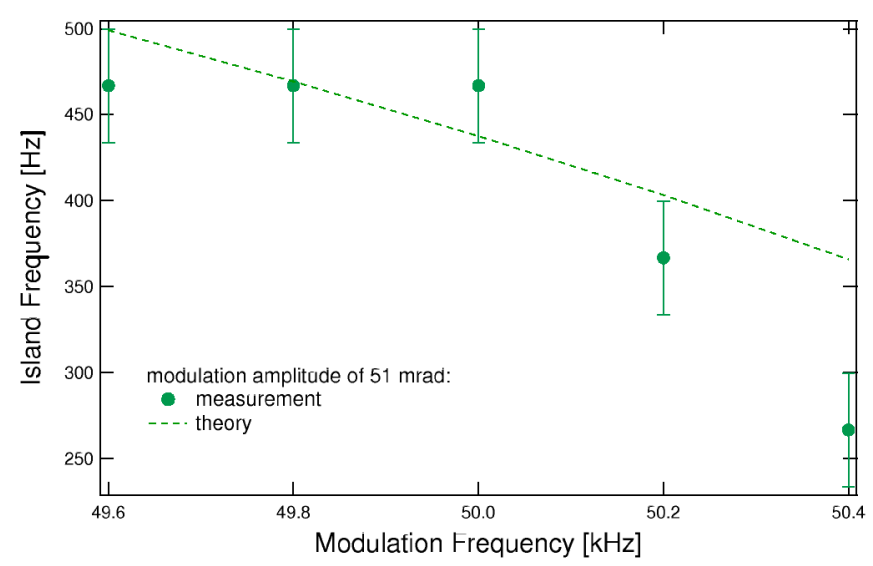

FIG. 17. (Color) Island frequency as a function of modulation frequency for a gap voltage of $460 \mathrm{kV}$.

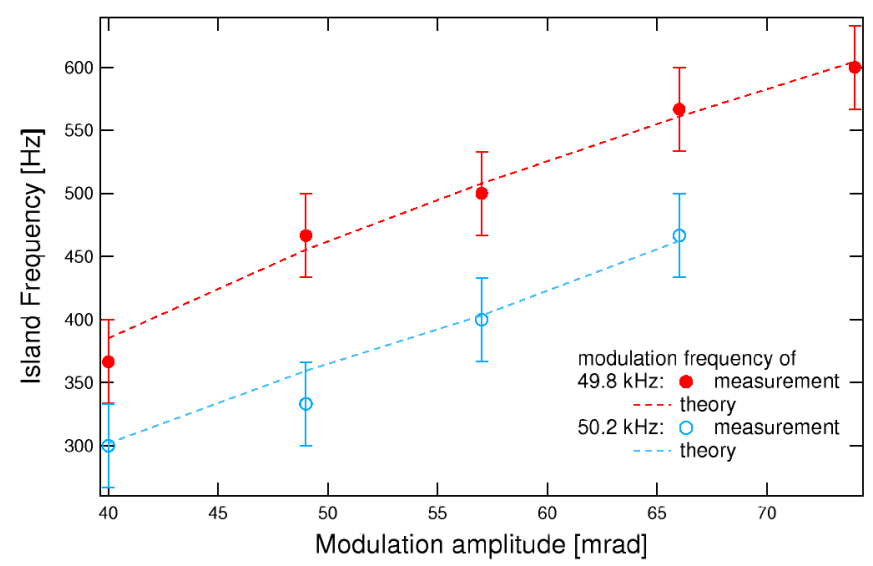

FIG. 18. (Color) Island frequency as a function of modulation amplitude for a gap voltage of $460 \mathrm{kV}$. shows good agreement between theory and experimental data. The error bars are relatively large due to the difficulties of measuring the damping time.

\section{Measurement of the island tune}

A fast-Fourier-transform of the damping time measurement data also shows a lower sideband of the line corresponding to half of the modulation frequency. This sideband, shown in Fig. 16, corresponds to the island tune [Appendix B]. Figures 17 and 18 show the measured dependence of the island frequency with modulation parameters and the corresponding theoretical curves (31), which are in good agreement.

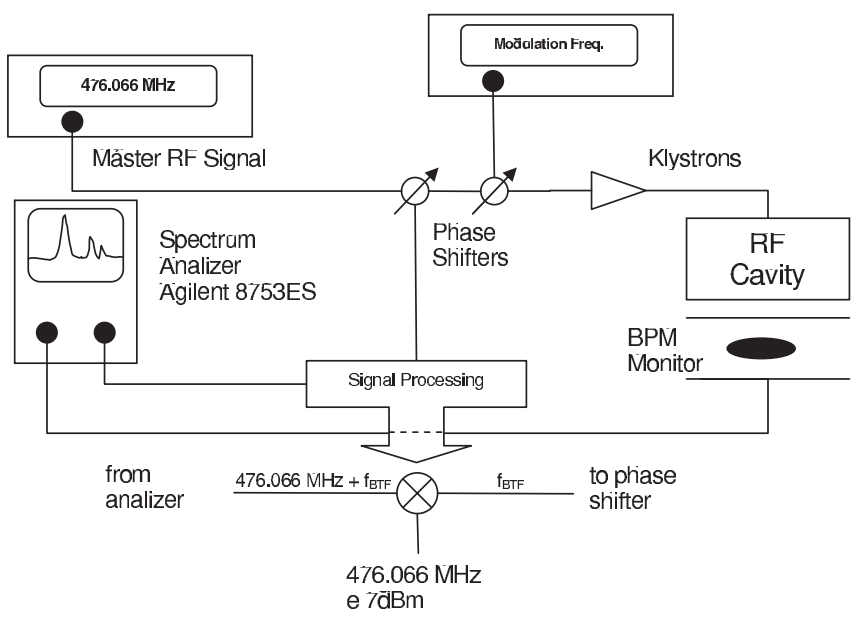

FIG. 19. Experimental setup used for measuring the BTF.
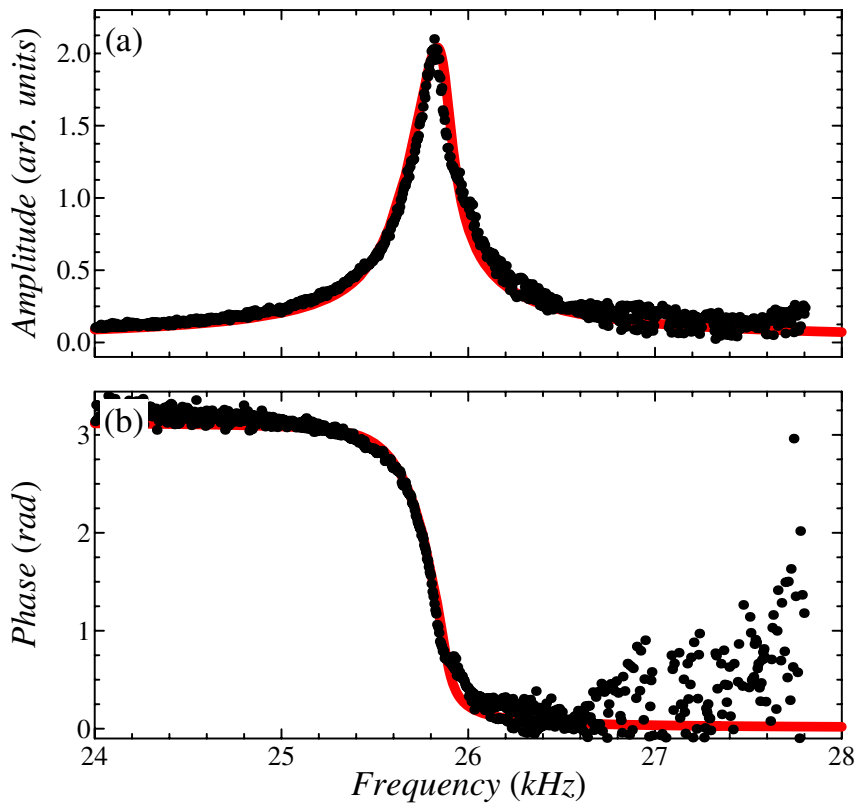

FIG. 20. (Color) Measurement of BTF for a single bunch without phase modulation for a gap voltage of $500 \mathrm{kV}$. The dots correspond to data points and the full red line to theory. 

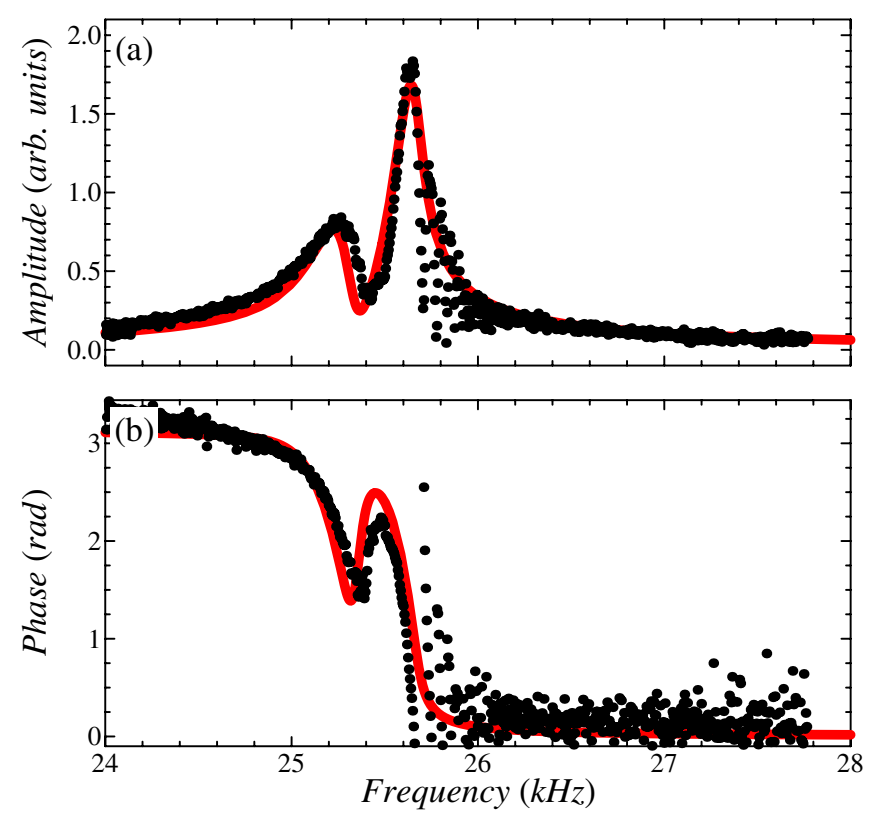

FIG. 21. (Color) Measurement of BTF for a single bunch, for a gap voltage of $500 \mathrm{kV}$, with phase modulation using the following parameters: $f_{m}=51 \mathrm{kHz}, A_{m}=35 \mathrm{mrad}$, and $N_{c}=65 \%$. The dots correspond to data points and the full red line to theory.

\section{Measurement of the BTF}

The experimental setup used to measure the BTF is shown in Fig. 19. We use a network analyzer to excite a dipolar oscillation on a low current single bunch and measure the beam response in amplitude and phase using a button pickup.

The results for a set of measurements with phase modulation turned on and off are shown in Figs. 20 and 21, respectively. The dots are the experimental data and the full line comes from the theoretical model. The result of those measurements indicates that the phase modulation pro-

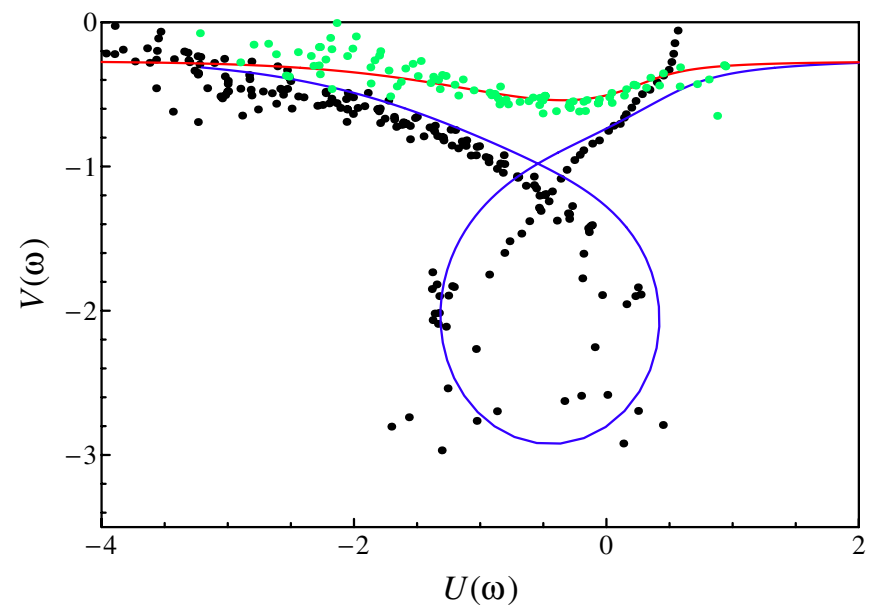

FIG. 22. (Color) Stability diagram of a single bunch with (black dots) and without (green dots) phase modulation and the respective theoretical curves. duces an increase in frequency spread within the bunch since the width in the amplitude response it related to this spread.

Figure 22 shows the stability diagram for the same set of measurements above [Figs. 20 and 21] and the corresponding theoretical curves. The green dots and red line correspond to the case without modulation, whereas the black dots and blue line correspond to a phase modulation amplitude of $35 \mathrm{mrad}$ and frequency of $51 \mathrm{kHz}$. Note that the stable area is effectively increased when phase modulation is turned on and also that some features, such as the loop predicted by the model, are present in the experimental data.

\section{DISCUSSION AND CONCLUSIONS}

We have shown experimental and theoretical evidence that, when rf phase modulation on the second harmonic of the synchrotron frequency is used, the available stable area to the electron beam is effectively increased and that the mechanism responsible for this enhancement in stability is an increase in the incoherent frequency spread which, via Landau damping, helps to alleviate unstable CBMs. We also simulate and calculate the island formation and perform measurements that indicate that these structures are indeed formed in the longitudinal phase space.

Our simulations also indicate that the lengthening of the bunches which results from phase modulation (an effect which has already been used at several laboratories as a tool to increase Touschek lifetime of intense bunches) cannot explain by itself the stabilization of CBMs that is observed when phase modulation is applied. In fact, only when bunches with internal structure (and corresponding frequency spread) are included in the simulations do we observe the intense damping of CBMs that is obtained in experiments.

Finally, the model developed to describe the BTF measurements presents all interesting features of the experimental results, namely, the double peaks and a second phase jump (which shows up as the loop of the stability diagram), although the island population still remains as a free parameter. We hope to be able to access this quantity in the future by using a streak camera.

We have been using rf phase modulation since 2004 to stabilize the electron beam of the LNLS storage ring. Although in the Brazilian machine the overall lifetime increase (we observed a lifetime increase of 30\%) was not as great as the one observed in other laboratories [911], the orbit fluctuation caused by the coupling of the beam with a HOM of the rf cavities was successfully suppressed improving the beam quality for users.

\section{ACKNOWLEDGMENTS}

We would like to express deep gratitude to the LNLS RF and diagnostics group whose support were crucial for the 
successful completion of this work. This work was completed with the support of FAPESP (Fundação de Apoio a Pesquisa do Estado de São Paulo).

\section{APPENDIX A: LONGITUDINAL HAMILTONIAN AROUND THE SECOND HARMONIC RESONANCE}

Here we present the detailed derivation of the Hamiltonian with phase modulation. Beginning with the Hamiltonian (4) and changing variables to

$$
\phi=\sqrt{2 \tilde{J}} \cos \tilde{\psi} \quad \text { and } \quad \delta=-\sqrt{2} \tilde{J} \sin \tilde{\psi},
$$

$$
\begin{aligned}
H(\tilde{J}, \tilde{\psi})= & \omega_{s} \tilde{J} \sin ^{2} \tilde{\psi}-\omega_{s} \sqrt{2 \tilde{J}} \tan \bar{\phi}_{s} \cos \tilde{\psi} \\
& +\omega_{s} \tan \bar{\phi}_{s} \sin \left[\sqrt{2 \tilde{J}} \cos \tilde{\psi}+A_{m} \sin \omega_{m} t\right] \\
& -\omega_{s} \cos \left[\sqrt{2} \tilde{J} \cos \tilde{\psi}+A_{m} \sin \omega_{m} t\right] .
\end{aligned}
$$

Expanding the terms in $\sin \tilde{\psi}$ and $\cos \tilde{\psi}$ using Bessel functions and restricting our analysis to even resonances close to the second harmonic of the synchrotron frequency $\left(\omega_{m} \approx 2 \omega_{s}\right)$, we can simplify the equation above to

we get

$$
\begin{aligned}
H(\tilde{J}, \tilde{\psi})= & \left(\omega_{s}-\frac{\omega_{s}}{2} \cos 2 \tilde{\psi}\right) \tilde{J}-\frac{\omega_{s} \tilde{J}^{2}}{16}-\omega_{s} \tan \bar{\phi}_{s} \sqrt{2 \tilde{J}} \cos \tilde{\psi}+\omega_{s} \tan \bar{\phi}_{s} \sqrt{2 \tilde{J}} \sin \left(\omega_{m} t\right) \tilde{J}_{0}(\sqrt{2 \tilde{J}}) \\
& -2 \omega_{s} \sum_{k=1}^{\infty}(-1)^{k} \tilde{J}_{2 k}(\sqrt{2 \tilde{J}}) \cos (2 k \tilde{\psi})+2 \omega_{s} \tan \bar{\phi}_{s} \sum_{k=0}^{\infty}(-1)^{k} \tilde{J}_{2 k+1}(\sqrt{2 \tilde{J}}) \cos [(2 k+1) \tilde{\psi}] \\
& -\omega_{s} \tan \bar{\phi}_{s} A_{m} \tilde{J}_{2}(\sqrt{2 \tilde{J}}) \sin \left(\omega_{m} t-2 \tilde{\psi}\right) .
\end{aligned}
$$

The new Hamiltonian is still time dependent and in order to eliminate this dependence we can perform a canonical transformation using the following generator function:

$$
F_{2}(J, \psi)=\left(\tilde{\psi}-\frac{\omega_{m} t}{2}-\frac{\pi}{4}\right) J
$$

so that the new coordinates in a rotating reference frame are

$$
\tilde{J}=\frac{\partial F_{2}}{\partial \psi}=J \quad \text { and } \quad \psi=\frac{\partial F_{2}}{\partial J}=\tilde{\psi}-\frac{\omega_{m} t}{2}-\frac{\pi}{4}
$$

and the Hamiltonian is

$$
K=H(J, \psi)+\frac{\partial F_{2}}{\partial t}=H(J, \psi)-\frac{\omega_{m}}{2} J
$$

performing a time average, the fast oscillation terms will vanish and we find that

$$
\langle K\rangle_{t}=\left(\omega_{s}-\frac{\omega_{m}}{2}\right) J-\frac{\omega_{s} J^{2}}{16}+\frac{\omega_{s} \epsilon J}{4} \cos 2 \psi
$$

which is the Hamiltonian around the second harmonic synchrotron resonance.

\section{APPENDIX B: CALCULATION OF THE ISLAND FREQUENCY IN THE LABORATORY REFERENCE FRAME}

In the rotating reference frame the motion of the particles in the islands can be described by

$$
\phi^{\prime}=r_{0} \cos \left(\omega^{\prime} t\right)+x_{0} \quad \text { and } \quad \delta^{\prime}=-r_{0} \sin \left(\omega^{\prime} t\right),
$$

where $r_{0}$ is the amplitude of the oscillations around the stable fixed point, $x_{0}$ is the stable fixed point coordinate in phase space, and $\omega^{\prime}=\sqrt{A B}$ is either the lateral island frequency or the central island frequency. To go from the rotating frame (which rotates clockwise with respect to the lab frame) to the laboratory reference frame, it is necessary to transform the above set of equations using the following rotation matrix:

$$
\mathcal{R}\left(\omega_{m} t / 2\right)=\left[\begin{array}{cc}
\cos \left(\frac{\omega_{m} t}{2}\right) & \sin \left(\frac{\omega_{m} t}{2}\right) \\
-\sin \left(\frac{\omega_{m}}{2}\right) & \cos \left(\frac{\omega_{m} t}{2}\right)
\end{array}\right]
$$

and the new particle coordinates are

$$
\left(\begin{array}{l}
\phi \\
\delta
\end{array}\right)=\mathcal{R}\left(\omega_{m} t / 2\right)\left(\begin{array}{c}
\phi^{\prime} \\
\delta^{\prime}
\end{array}\right),
$$

or writing explicitly for each coordinate

$$
\begin{gathered}
\phi=r_{0} \cos \left[\left(\omega^{\prime}+\frac{\omega_{m}}{2}\right) t\right]+x_{0} \cos \left(\frac{\omega_{m} t}{2}\right), \\
\delta=r_{0} \sin \left[\left(\omega^{\prime}+\frac{\omega_{m}}{2}\right) t\right]+x_{0} \sin \left(\frac{\omega_{m} t}{2}\right) .
\end{gathered}
$$

To understand this set of equations, we have first to know whether particles in the islands rotate clockwise or counterclockwise. Taking the harmonic part of the Hamiltonian (21) expanded around each stable fixed point, we find that

$$
\frac{d \phi^{\prime}}{d t}=A \delta^{\prime} \quad \text { and } \quad \frac{d \delta^{\prime}}{d t}=B \phi^{\prime},
$$

so that if $A>0$ and $B>0$ the particles rotate clockwise and if $A<0$ and $B<0$ the particles rotate counterclockwise. 
Calculating the coefficients $A$ and $B$ for our parameters, we find that particles in the central island rotate clockwise and particles in the lateral islands rotate counterclockwise. Using this result we can write the particle equations in the lab reference frame:

$$
\begin{aligned}
\phi_{i} & =r_{0} \cos \left[\left(\frac{\omega_{m}}{2}-\omega_{i}\right) t\right]+x_{0} \cos \left(\frac{\omega_{m} t}{2}\right), \\
\delta_{i} & =r_{0} \sin \left[\left(\frac{\omega_{m}}{2}-\omega_{i}\right) t\right]+x_{0} \sin \left(\frac{\omega_{m} t}{2}\right),
\end{aligned}
$$

and

$$
\begin{gathered}
\phi_{c}=r_{0} \cos \left[\left(\frac{\omega_{m}}{2}+\omega_{c}\right) t\right], \\
\delta_{c}=r_{0} \sin \left[\left(\frac{\omega_{m}}{2}+\omega_{c}\right) t\right],
\end{gathered}
$$

for the lateral islands and central island, respectively.

From the equations above, we note that when measuring in the laboratory reference frame the particles respond to an external excitation with three different frequencies: a narrow line at $\omega_{m} / 2$, which is related to the external phase modulation, and a broad line at $\omega_{m} / 2-\omega_{i}$ and $\omega_{m} / 2+$ $\omega_{c}$ that corresponds to the particles incoherent revolution frequencies around each stable fixed point.

[1] M. Ellison et al., Phys. Rev. Lett. 70, 591 (1993).

[2] M. Syphers et al., Phys. Rev. Lett. 71, 719 (1993).

[3] H. Huang et al., Phys. Rev. E 48, 4678 (1993).

[4] D. Li, M. Ball, B. Brabson, J. Budnick, D. D. Caussyn, A. W. Chao, J. Collins, V. Derenchuk, S. Dutt, G. East, M. Ellison, D. Friesel, B. Hamilton, H. Huang, W. P. Jones, S. Y. Lee, M. G. Minty, S. Nagaitsev, K. Y. Ng, X. Pei, T. Sloan, M. Syphers, L. Teng, Y. Wang, Y. T. Yan, and P. L. Zhang, Phys. Rev. E 48, R1638 (1993).

[5] D. Li et al., Nucl. Instrum. Methods Phys. Res., Sect. A 364, 205 (1995).
[6] M.H. Wang et al., in Proceedings of the Particle Accelerator Conference, New York, 1999, pp. 2837-2839.

[7] Y. Zhang and S. Y. Lee, in Proceedings of the Particle Accelerator Conference, Chicago, 2001, pp. 1726-1728.

[8] C.J. Bochetta et al., in Proceedings of the Particle Accelerator Conference, Vancouver, 1997, pp. 829-831.

[9] P. Kuske, in Proceedings of the European Particle Accelerator Conference, Stockholm, 1998, pp. 12971299.

[10] Yu. Senichev et al., in Proceedings of the European Particle Accelerator Conference, Stockholm, 1998, pp. 1339-1341.

[11] S. Sakanaka, M. Izawa, T. Mitsuhashi, and T. Takahashi, Phys. Rev. ST Accel. Beams 3, 050701 (2000).

[12] M.H. Wang et al., in Proceedings of the Particle Accelerator Conference, New York, 1999, pp. 1487-1489.

[13] J. M. Byrd, W. H. Cheng, and F. Zimmermann, Phys. Rev. E 57, 4706 (1998).

[14] F. Orsini and A. Mosnier, Phys. Rev. E 61, 4431 (2000).

[15] L. Lin, L. Jahnel, and P. F. Tavares, in Proceedings of the European Particle Accelerator Conference, Berlin, 1992, p. 450.

[16] R.H.A. Farias, C. Pardine, and P.F. Tavares, in Proceedings of the European Particle Accelerator Conference, Paris, 2002, pp. 2148-2150.

[17] R.H.A. Farias et al., in Proceedings of the European Particle Accelerator Conference, Lucern, 2004, pp. 6192-6194.

[18] M. Svandrlik et al., in Proceedings of the European Particle Accelerator Conference, London, 1994, pp. 2762-2764.

[19] S. Sakanaka and T. Obina, Jpn. J. Appl. Phys. 40, 2465 (2001).

[20] L. D. Landau and E. M. Lifchitz, Mechanics (Éditions Mir, Moscou, 1966), 12th ed.

[21] S. Y. Lee, Accelerator Physics (World Scientific, Singapore, 1999), p. 234.

[22] A. W. Chao, Physics of Collective Beam Instabilities in High Energy Accelerators (John Wiley \& Sons, New York, 1993).

[23] J. M. Byrd, Part. Accel. 57, 159 (1997). 